\title{
Pore pressure and stress distribution analysis around an inclined wellbore in a transversely isotropic formation based on the fully coupled chemo-thermo-poroelastic theory
}

\author{
Wenke Cao ${ }^{a}$, Jingen Deng ${ }^{a}$, Wei Liu ${ }^{a}{ }^{\text {, }}$, Baohua $\mathrm{Yu}^{\mathrm{a}}$, Qiang Tan ${ }^{\mathrm{a}}$, Liu Yang ${ }^{\mathrm{b}}$, Yang $\mathrm{Li}^{\mathrm{a}}$, \\ Jiajia Gao ${ }^{a}$ \\ a State Key Laboratory of Petroleum Resources and Prospecting, China University of Petroleum, Beijing 102249, China \\ ${ }^{\mathrm{b}}$ Key Laboratory for Mechanics in Fluid Solid Coupling Systems, Institute of Mechanics, Chinese Academy of Sciences, 100190 Beijing, China
}

\section{A R T I C L E I N F O}

\section{Article history:}

Received 10 October 2016

Received in revised form

3 January 2017

Accepted 3 February 2017

Available online 12 February 2017

\section{Keywords:}

Chemo-thermo-poroelastic

Fully coupled

Finite element

Wellbore stability

Shale

\begin{abstract}
A B S T R A C T
Wellbore instability is a widespread problem when drilling in shale formations, particularly with waterbased drilling fluid. The main reason for the occurrence of wellbore instability is that stress concentration and pore pressure redistribution occur around the wellbore once a hole is drilled; current studies show that the drilling fluid temperature and solute mass fraction play important roles during the process. In this work, a non-linear fully coupled chemo-thermo-poroelastic finite element model is developed to quantitatively access thermal and chemical effects on time-dependent pore pressure and effective stresses; in addition, material constant sensitivity analysis of an inclined well drilled in a transversely isotropic formation is presented. The results reveal the following: fluid transfer is greatly affected by thermal and chemical osmosis, the lower temperature and higher solute mass fraction of the drilling fluid contribute to decreasing the pore pressure and are beneficial for wellbore stability, and thermal parameters (such as thermal osmosis coefficient and thermal diffusivity) and chemical parameters (such as reflection coefficient and solute diffusion coefficient) have high effects on the pore pressure and effective stresses. Anisotropy ratio analysis of the material constants indicates that the pore pressure and effective stresses are very sensitive to Young's modulus and the permeability ratio, but are not sensitive to Poisson's ratio. Therefore, the developed coupled chemo-thermo-poroelastic theory illustrates that optimization of the reduction of the drilling fluid temperature while maintaining a high solute mass fraction could enhance wellbore stability.
\end{abstract}

(C) 2017 Elsevier B.V. All rights reserved.

\section{Introduction}

Wellbore instability, such as collapse and fracture, is a challenging problem when drilling in a shale formation. Because shale formations are originally chemo-thermo-hydro-mechanical equilibrium systems prior to drilling, once a wellbore is drilled, the equilibrium is broken, and thus, the pore pressure and effective stress redistribute via fluid flow and wellbore deformation. Many authors have investigated the pore pressure and distribution of effective stresses in the vicinity of a wellbore wall from mechanical, hydraulic, thermal and chemical aspects based on different theories.

\footnotetext{
* Corresponding author.

E-mail address: 276914999@qq.com (W. Liu).
}

Biot (1941) poroelastic theory assumed rock to be a porous media, including an elastic solid matrix and saturated pore fluid; the coupling deformation of solids and fluids could occur as a result of a disturbed pressure. Subsequently, a fully coupled poroelastic theory (Detournay and Cheng, 1988) was introduced to analyze the pore pressure and stress distribution; these variables were demonstrated to be time dependent with pore fluid transport. In addition to the hydraulic pressure difference between drilling fluid and pore fluid, chemical and thermal gradients also contribute to water flowing into or out of the formation. Many authors (Chenevert and Dewan, 2001; F. K. Mody and Hale, 1993a,b; Sherwood, 1993; Sherwood and Craster, 2000) have studied the chemical osmosis effect on fluid flow. Ekbote and Abousleiman (2006) investigated fully coupled chemo-poroelastic problem by extending Detournay and Sherwood's work and eventually obtained analytical solutions that are suitable for isotropic and 
transversely isotropic formations; Ghassemi et al. (1999); Ghassemi and Diek (2002, 2003) presented another set of constitutive equations for the chemo-poroelastic problem based on Heidug and Wong's work (1996) and also provided analytical solutions. Moreover, considerable research studies have been conducted by incorporating thermal effects with the poroelastic theory: Palciauskas and Domenico (1982) first developed thermoporoelastic constitutive equations, and analytical solutions that were provided by Wang and Papamichos (1994) and Wang and Dusseault (2003) suggested that both heat conduction and convection should be considered to analyze the poroelasticity problem.

Previous researchers have also studied the combination chemical potential and thermal osmosis effects on wellbore stability, for example, two sets of coupling theories were established and analytical solutions were provided independently by Ekbote (2002) and Ghassemi et al. (2009), Tao et al. (2005); Tao and Ghassemi (2006).

Because an underground formation is always regarded as a transversely isotropic medium, Biot's theory has been extended to account for material anisotropy based on chemo-poroelastic and thermo-poroelastic theory, and a set of analytical solutions were given; however, these analytical solutions are only suitable for the wellbore, which is vertical with a transverse plane. To overcome the limitations of analytic solutions, numerical methods were applied by some authors. Cui et al. (1996, 1997) established FEM equations for coupled anisotropic and non-linear poroelasticity, and the problem of inclined boreholes was investigated by the threedimensional (3D) FEM model and generalized plane strain FEM model. Ghassemi et al. (2001) and Ghassemi and Zhang (2004) applied the boundary element method to determine the distribution of the stress and pore pressures of a well in a poroelastic medium. Gomar et al. (2015, 2014a, 2014b) adopted a twodimensional finite element method to study wellbore stability based on the thermo-poroelastic theory, which takes into account conductive and convective transport processes. Roshan and Aghighi (2012) developed a finite element model based on chemo-poroelascity and found that a threshold in the formation permeability exists that can determine when the convection influence cannot be ignored. These numerical studies and analytical solutions are based on linear theory. Zhou and Ghassemi (2009) presented a two-dimensional finite element model based on nonlinear chemo-poro-thermoelasticity that is applicable for an isotropic formation.

In this paper, a three-dimensional finite element model, which is based on the non-linear fully coupled chemo-thermo-poroelastic theory and also available for a directional wellbore stability analysis in a transversely isotropic shale formation, is developed by extending Zhou and Ghassemi's work (2009). The numerical model is first verified by comparison with the analytical results of chemothermo-poroelasticity, and then, sensitivity analysis is conducted to assess the effect of the temperature and solute mass fraction difference between drilling fluid and pore fluid; in addition, thermal and chemical parameters as well as the sensitivity of the material constant anisotropy ratios are investigated.

\section{Coupled chemo-thermo-poroelastic theory}

The chemo-thermo-poroelastic model is formulated by extending Boit's poroelastic model to incorporate chemical and thermal effects; the details of the theoretical development is given by Diek et al. (2011).

(1) Constitutive equations

The general constitutive equations with regard to stress and the fluid volume for the non-linear chemo-thermo-poroelastic model can be written in the following form, where a tension positive convention is adopted in this paper and bold fonts represent tensors or vectors in equations.

$$
\begin{aligned}
& \boldsymbol{\sigma}=\mathbf{D} \boldsymbol{\varepsilon}-\boldsymbol{\alpha}^{\prime} p+\chi C^{S}-\gamma_{1} T \\
& \zeta=\boldsymbol{\alpha}^{T} \boldsymbol{\varepsilon}+\beta p+\chi^{\prime} C^{S}-\gamma_{2} T
\end{aligned}
$$

The coefficients in the above equations are given below:

$\boldsymbol{\alpha}^{\prime}=\boldsymbol{\alpha}-\frac{M^{S} \omega_{0}}{\bar{\rho}_{f} R T C^{D}} \cdot \mathbf{I} ; \quad \beta=\frac{\mathbf{m}^{T} \boldsymbol{\alpha} / 3-\phi}{K_{S}}+\frac{\phi}{K_{f}}+\frac{\omega_{0}\left(\mathbf{m}^{T} \boldsymbol{\alpha} / 3-1\right) M^{S}}{\bar{\rho}_{f} K R C^{D} T}$

$$
\begin{aligned}
\boldsymbol{\chi} & =\left(1-\frac{C^{S}}{C^{D}}\right) \frac{\omega_{0}}{C^{S}} \cdot \mathbf{I} ; \quad \chi^{\prime}=\chi^{T} \frac{\boldsymbol{\alpha}-\mathbf{m}}{K} \\
\gamma_{1} & =\mathbf{D} \boldsymbol{\alpha}_{m}+\frac{s_{0} \omega_{0} M^{S}}{R T C^{D}} \cdot \mathbf{I} ; \quad \gamma_{2} \\
& =\boldsymbol{\alpha}^{T} \boldsymbol{\alpha}_{m}+\left(\alpha_{f}-\mathbf{m}^{T} \boldsymbol{\alpha}_{m}\right) \phi+\frac{s_{0} \omega_{0}\left(\mathbf{m}^{T} \boldsymbol{\alpha} / 3-1\right) M^{S}}{K R T C^{D}}
\end{aligned}
$$

$\mathbf{m}=[1,1,1,0,0,0]^{T}$

\section{(2) Transport equations}

Shale's permeability is so low that chemical potential gradient and thermal gradient effect can't be neglected (Diek et al., 2011) comparing to hydraulic transport, thermal gradient can drive fluid molecules transport from higher temperature to lower temperature side, so pore fluid flux is assumed to be caused by the gradient of pressure, chemical osmosis and thermal osmosis, solute flux is determined by solute and thermal diffusion, and heat transfer is merely controlled by the temperature gradient. Thus, the transport equations are given by

$\mathbf{J}^{f}=-\kappa\left(\nabla p-L_{D} \nabla C^{S}\right)-\mathbf{K}_{T} \nabla T$

$\mathbf{J}^{S}=-\mathbf{D}_{S} \nabla C^{S}-\mathbf{D}_{T} \nabla T$

$\mathbf{J}^{T}=-\mathbf{k}_{T} \nabla T$

where $L_{D}=\frac{\Re \bar{\rho}_{f} R T}{M^{S} C^{S} C^{D}}$

(3) Momentum balance equation

$\nabla \cdot \sigma=0$

(4) Conservation equations

Conservation equations of fluid, solute mass and temperature are given as

$\frac{\partial \varsigma}{\partial t}+\nabla \cdot \mathbf{f}^{f}=0$

$\phi \frac{\partial C^{s}}{\partial t}+\nabla \cdot \mathbf{J}^{s}=0$ 
$\rho C_{v} \frac{\partial T}{\partial t}+\nabla \cdot \mathbf{J}^{T}=0$

\section{Coordinate transformation}

All of the material constants included in the aforementioned equations, such as the elastic stiffness tensor $\mathbf{D}$ (Young's modulus and Poisson's ratio), Biot coefficient $\alpha$, permeability $\mathbf{k}$ and in-situ stress $\sigma$, are tensors defined in the borehole Cartesian coordinate system, but generally, material constants are given in material principle directions and in-situ stresses are given in vertical and horizontal directions. To obtain these parameters in a borehole coordination system, a complex transformation must be performed; thus, five coordinate systems are defined to clearly describe these relationships: a global coordinate system (GCS), insitu stress coordinate system (ICS), transversely isotropic formation coordinate system (FCS), borehole coordinate system (BCS) and borehole cylindrical coordinate system (CCS). Fig. 1 shows the coordinate systems and their relationship; because it is typically assumed that overburden stress is one of the principle in-situ stresses, $\beta_{s}=0$.

The in-situ stress $\sigma$ of BCS should be transformed from ICS to GCS and then to BCS, whereas material constants $\boldsymbol{\alpha}, \mathbf{k}$ should be transformed from FCS to GCS and then to BCS. Every transformation step includes two sequential steps; the transformation of GCS to BCS can be used as an example: rotate the original $X_{g}-Y_{g}-Z_{g}$ axes by well azimuth angle $\beta_{b}$ around the z-axes to obtain the new axes, and then, rotate the new axes by the well inclination angle $\alpha_{b}$ to obtain the $X_{b}-Y_{b}-Z_{b}$ axes. This transformation corresponds to the following matrix

$\lambda_{g 2 b}=\left[\begin{array}{ccc}\cos \left(\alpha_{h}\right) & & -\sin \left(\alpha_{h}\right) \\ & 1 & \\ \sin \left(\alpha_{h}\right) & & \cos \left(\alpha_{h}\right)\end{array}\right]\left[\begin{array}{ccc}\cos \left(\beta_{h}\right) & \sin \left(\beta_{h}\right) & \\ -\sin \left(\beta_{h}\right) & \cos \left(\beta_{h}\right) & \\ & & 1\end{array}\right]$

Thus, the in-situ stress in borehole coordinate system is given by

$\sigma_{b}=\lambda_{g 2 b} \sigma_{g} \lambda_{g 2 b}^{T}$

Other material constants can be obtained by a similar method. Moreover, the elastic stiffness tensor under BCS should also be transformed from FCS; the elastic stiffness tensor of transversely isotropic formation $\mathbf{D}_{f}$ is given in matrix form as

$$
\mathbf{D}_{f}=\left[\begin{array}{llllll}
D_{11} & D_{12} & D_{13} & & & \\
D_{12} & D_{11} & D_{13} & & & \\
D_{13} & D_{13} & D_{33} & & & \\
& & & G & & \\
& & & & G^{\prime} & \\
& & & & & G^{\prime}
\end{array}\right]
$$

where

$D_{11}=\frac{E_{h}\left(E_{v}-E_{h} \nu_{v}^{2}\right)}{\left(1+\nu_{h}\right)\left(E_{v}-E_{v} \nu_{h}-2 E_{h} \nu_{v}^{2}\right)}$

$D_{12}=\frac{E_{h}\left(E_{v} \nu_{h}+E_{h} \nu_{v}^{2}\right)}{\left(1+\nu_{h}\right)\left(E_{v}-E_{v} \nu_{h}-2 E_{h} \nu_{v}^{2}\right)}$

$D_{13}=\frac{E_{h} E_{v} \nu_{v}}{E_{v}-E_{v} \nu_{h}-2 E_{h} \nu_{v}^{2}} ; \quad D_{33}=\frac{E_{v}^{2}\left(1-\nu_{h}\right)}{E_{v}-E_{v} \nu_{h}-2 E_{h} \nu_{v}^{2}}$

Then, the elastic stiffness tensor in the borehole coordinate system is given by

$\mathbf{D}_{b}=\mathbf{T}_{f 2 b} \times \mathbf{D}_{f} \times \mathbf{T}_{f 2 b}^{T}$

The detailed transformation method and transformation matrix for the elastic stiffness tensor are presented by Lekhnitskii (1977).

\section{Finite element formula}

To easily simulate the initial stress and pore pressure of formation and apply the boundary condition, the three-dimensional model is established (Fig. 2); the model geometry comprises wellbore and formation; the wellbore radius is $0.1 \mathrm{~m}$, the length and width of the model are both $2 \mathrm{~m}$, and the height is $4 \mathrm{~m}$. Twentynode hexahedron quadratic elements are used for the solid displacement $\mathbf{u}$, and eight-node hexahedron elements are used for the pore pressure $p$, solute mass fraction $C^{S}$ and temperature $T$. In addition, the mesh is refined around the borehole to obtain more accurate results.

The initial conditions and boundary conditions of the pore pressure, solute mass fraction and temperature are required:

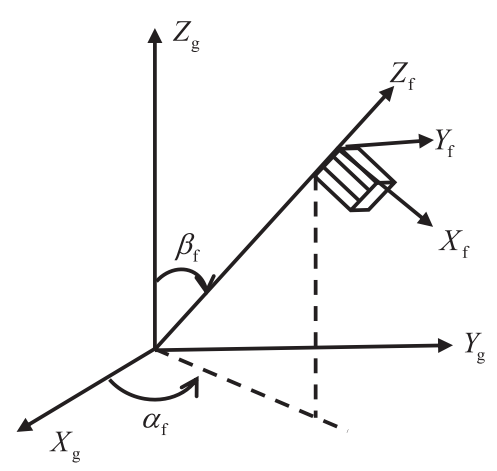

(a)

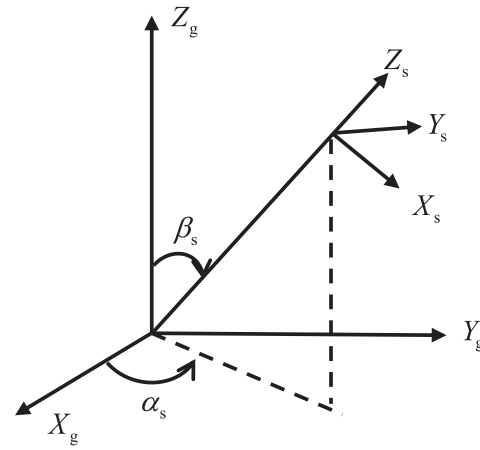

(b)

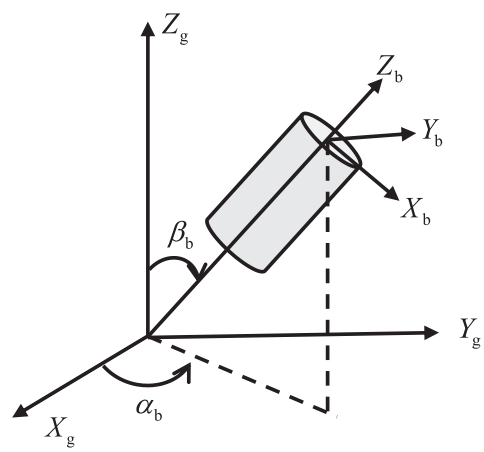

(c)

Fig. 1. Coordinate transformation systems: (a) relationship between GCS $\left(X_{g}-Y_{g}-Z_{g}\right)$ and FCS $\left(X_{f}-Y_{f}-Z_{f}\right)$, (b) relationship between GCS $\left(X_{g}-Y_{g}-Z_{g}\right)$ and ICS $\left(X_{s}-Y_{s}-Z_{s}\right)$, (c) relationship between GCS $\left(X_{g}-Y_{g}-Z_{g}\right)$ and BCS $\left(X_{b}-Y_{b}-Z_{b}\right)$. 


$$
\left\{\begin{array}{l}
p(r, t<0)=p\left(r_{\infty}, t \geq 0\right)=p_{0} \\
C^{S}(r, t<0)=C^{S}\left(r_{\infty}, t \geq 0\right)=C_{0}^{S} \\
T(r, t<0)=T\left(r_{\infty}, t \geq 0\right)=T_{0} \\
p\left(r_{W}, t \geq 0\right)=p_{m} \\
C^{S}\left(r_{W}, t \geq 0\right)=C_{m}^{S} \\
T\left(r_{W}, t \geq 0\right)=T_{m}
\end{array}\right.
$$

To transform these equations to finite element formulation, the variables of displacement $u$, pore pressure $p$, solute mass fraction $C^{S}$ and temperature $T$ are discretized as follows:

$$
\left\{\begin{array}{l}
u=\mathbf{N}_{u} \cdot \mathbf{U} \\
p=\mathbf{N}_{p} \cdot \mathbf{P} \\
C^{S}=\mathbf{N}_{C^{s}} \cdot \mathbf{C}^{S} \\
T=\mathbf{N}_{T} \cdot \mathbf{T}
\end{array}\right.
$$

where $\mathbf{N}_{u}, \mathbf{N}_{p}, \mathbf{N}_{C^{s}}, \mathbf{N}_{T}$ are the shape functions of displacement, pore pressure, solute mass fraction, and temperature, respectively; $\mathbf{U}, \mathbf{P}, \mathbf{C}^{\mathbf{S}}, \mathbf{T}$ are the vectors of the nodal values. A finite element method is constructed by substituting Eq. (22) into Eq. (1) through (13), and then, the variational method is used for time discretization; the finite element formulation of the field equation is eventually given by

$$
\begin{gathered}
\mathbf{K}_{1} \cdot \mathbf{U}+\mathbf{L}_{1} \cdot \mathbf{P}-\mathbf{W}_{1} \cdot \mathbf{C}^{s}+\mathbf{Q}_{1} \cdot \mathbf{T}=\mathbf{E}_{1} \\
-\mathbf{A}_{2} \cdot \dot{\mathbf{U}}+\mathbf{S}_{2} \cdot \dot{\mathbf{P}}+\mathbf{M}_{2} \cdot \dot{\mathbf{C}}^{s}-\mathbf{H}_{2} \cdot \dot{\mathbf{T}}+\mathbf{L}_{2} \cdot \mathbf{P}-\mathbf{W}_{2} \cdot \mathbf{C}^{s}+\mathbf{Q}_{2} \cdot \mathbf{T}=\mathbf{E}_{2} \\
\mathbf{M}_{3} \cdot \dot{\mathbf{C}}^{s}+\mathbf{W}_{3} \cdot \mathbf{C}^{s}+\mathbf{Q}_{3} \cdot \mathbf{T}=\mathbf{E}_{3} \\
\mathbf{H}_{4} \cdot \dot{\mathbf{T}}+\mathbf{Q}_{4} \cdot \mathbf{T}=\mathbf{E}_{4}
\end{gathered}
$$

The matrices $\mathbf{K}_{1}, \mathbf{L}_{1}, \mathbf{W}_{1}, \mathbf{Q}_{1}, \mathbf{E}_{1}, \mathbf{A}_{2}, \mathbf{S}_{2}, \mathbf{M}_{2}, \mathbf{H}_{2}, \mathbf{L}_{2}, \mathbf{W}_{2}, \mathbf{Q}_{2}, \mathbf{E}_{2}$, $\mathbf{M}_{3}, \mathbf{W}_{3}, \mathbf{Q}_{3}, \mathbf{E}_{3}, \mathbf{H}_{4}, \mathbf{Q}_{4}, \mathbf{E}_{4}$ are defined in the appendix; the finite element formula of the governing equations is obtained:

$$
\begin{gathered}
{\left[\begin{array}{cccc}
\mathbf{K}_{1} & \mathbf{L}_{1} & -\mathbf{W}_{1} & \mathbf{Q}_{1} \\
-\frac{\mathbf{A}_{2}}{\Delta t} & \frac{\mathbf{S}_{2}}{\Delta t}+\mathbf{L}_{2} & \frac{\mathbf{M}_{2}}{\Delta t}-\mathbf{W}_{2} & -\frac{\mathbf{H}_{2}}{\Delta t}+\mathbf{Q}_{2} \\
0 & 0 & \frac{\mathbf{M}_{3}}{\Delta t}+\mathbf{W}_{3} & \mathbf{Q}_{3} \\
0 & 0 & 0 & \frac{\mathbf{H}_{4}}{\Delta t}+\mathbf{Q}_{4}
\end{array}\right]\left[\begin{array}{c}
\mathbf{U} \\
\mathbf{P} \\
\mathbf{C}^{s} \\
\mathbf{T}
\end{array}\right]} \\
+\left[\begin{array}{ccc}
\frac{\mathbf{A}_{2}}{\Delta t} \mathbf{U}_{t}-\frac{\mathbf{S}_{2}}{\Delta t} \mathbf{P}_{t}-\frac{\mathbf{M}_{2}}{\Delta t} \mathbf{C}_{t}^{s}+\frac{\mathbf{H}_{2}}{\Delta t} \mathbf{T}_{t} \\
\\
& -\frac{\mathbf{M}_{3}}{\Delta t} \Delta \mathbf{C}_{t}^{s} \\
& -\frac{\mathbf{H}_{4}}{\Delta t} \Delta \mathbf{T}_{t}
\end{array}\right]=\left[\begin{array}{l}
\mathbf{E}_{1} \\
\mathbf{E}_{2} \\
\mathbf{E}_{3} \\
\mathbf{E}_{4}
\end{array}\right]
\end{gathered}
$$

where $\Delta t$ is time increment between two adjacent steps. Because Eq. (24) shows that the element stiffness matrix (first term of left part) is asymmetrical and the equation is a non-linear problem because $C^{S}$ and $T$ included in Eq. (24) are unknown, an iterative method must be used for each step to solve the problem. This coupled problem can be solved by writing a user-defined element (UEL) subroutine in ABAQUS/Standard (Hibbett et al., 1998). The coupled temperature-displacement step is used, a standard model is first built and the element includes three displacement degrees of freedom (x-displacement, y-displacement and z-displacement) and three temperature degrees of freedom, because UEL can modify the degree of freedom freely, then two of three temperature degrees of freedom are changed to be pore pressure and solute mass fraction. The Newton-Nicolson incremental-iterative method is adopted to solve the wellbore non-linear chemo-thermo-poroelastic problem. The analysis is divided into several incremental steps, and every incremental step is subdivided into several iteration steps. The nonlinear problem is then transferred into a linear problem for each iteration step.

\section{Verification of the finite element method}

Because there are no analytical solutions for the non-linear chemo-thermo-poroelastic problem, particularly for arbitrary directional well drilled in transversely isotropic formation, the finite element method results must be verified with the analytical solutions for linear chemo-thermo-poroelastic problems. Analytical solutions were presented by Abousleiman and Ekbote (2001) and Tao et al. (2005). The fluid and formation parameters are presented in Table 1; the well drilled in the isotropic formation is assumed to be vertical; the maximum in-situ stress direction is north; the initial in-situ stress, pore pressure, solute mass fraction and temperature are applied in the formation model; the drilling operation is simulated by removing the borehole part element and apply drilling fluid hydraulic pressure, pore pressure, solute mass fraction and temperature on the borehole wall instantaneously at $t=0$; and the transient magnitudes of solute mass traction and temperature included in governing equations are substituted by the mean values to obtain analytical solutions for the linear chemothermo-poroelastic problem.

Fig. 3 shows pore pressure distribution around the wellbore along the minimum in-situ stress direction for the linear thermoporoelastic problem at time steps of $60 \mathrm{~s}, 600 \mathrm{~s}$ and $6000 \mathrm{~s}$, results of the numerical model show good agreement with the analytical solutions. Because the drilling fluid temperature and solute mass faction are lower than that of formation, the lower solute mass fraction of the drilling fluid forces fluid into the formation and increases the pore pressure around the wellbore, in contrast, the direct cooling formation and fluid molecular diffusion brings down pore pressure, thus the combined hydraulic, chemical and thermal effects make the pore pressure distributions show an interest result around the wellbore.

\section{Sensitivity analysis}

Chemical and thermal loading on poroelasticity are investigated in this section, and sensitivity analyses on the chemical and thermal coefficients and material constant anisotropy ratio are conducted. The effective radial and tangential stresses are generally the minimum and maximum principle stresses for a vertical well, and both of them could be compared with the strength criterion to determine the shear failure and tensile failure zone of the well. Therefore, the pore pressure and effective radial and tangential stresses along the minimum in-situ stress direction are the main analysis objects in this paper. The input parameters for this study are summarized in Table 1.

\subsection{Effect of the temperature and thermal parameters}

To study the temperature effect of drilling fluid, the solute mass fraction of drilling fluid is kept the same as that of the pore fluid, and the well is vertical and perpendicular to the isotropy plane of formation. First, three cases $\left(T_{W}>T_{0}, T_{W}=T_{0}\right.$ and $\left.T_{W}<T_{0}\right)$ are considered, and the temperature difference for heating and cooling is $40 \mathrm{~K}$. It should be noticed that even the solute mass faction of drilling fluid and pore fluid are same, constitutive equations (Eq. (1) and (2)) illustrate the chemical effect (pore pressure and temperature coefficients $\boldsymbol{\alpha}^{\prime}, \beta, \gamma_{1}, \gamma_{2}$ include solute and solvent mass 


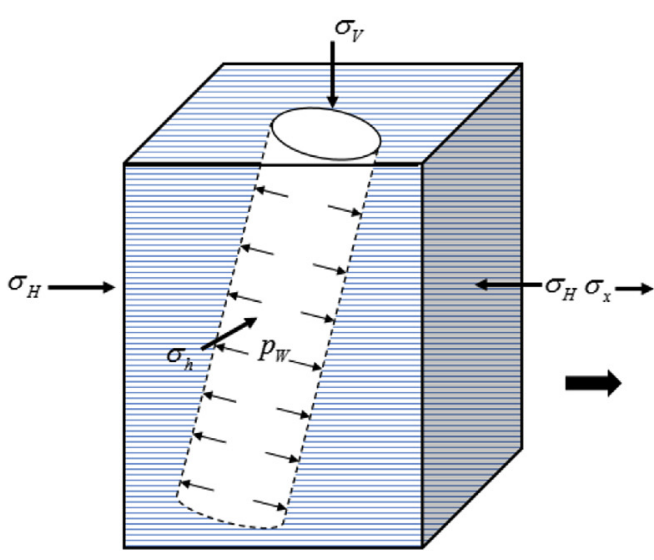

(a)

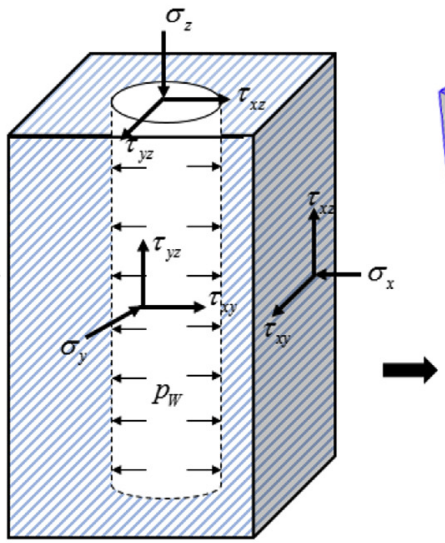

(b)

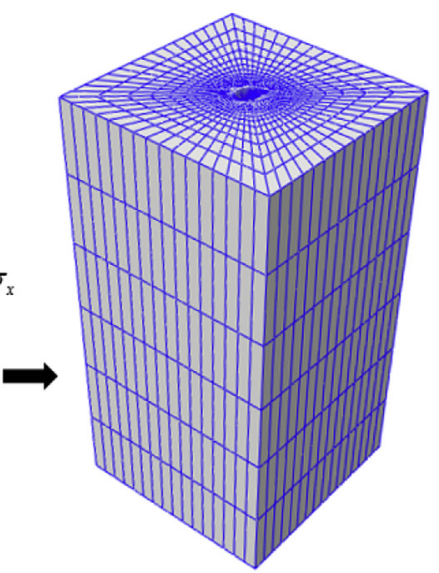

(c)

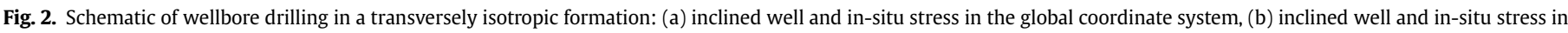
the borehole coordinate system for FEM, and (c) 20 node quadratic FEM mesh.

fraction $C_{S}, C_{D}$ ) still plays an important role in pore pressure and effective stress distribution. The pore pressure and effective radial and tangential stresses over a period of $60000 \mathrm{~s}$ ( 0.7 day) are presented in Figs. 4-6. It is a poroelastic problem without a thermal effect when $T_{W}=T_{0}$, which can be regarded as a reference for thermal effect analysis. Each figure is divided into two parts, so that the thermal effect can be observed and analyzed intuitively; the main part of the figure is the pore pressure or effective stress distribution with the radius, and the upper or lower right corner part

Table 1

Input data in the analysis.

\begin{tabular}{|c|c|}
\hline Parameter & Value \\
\hline Borehole radius, $r_{W}$ & 0.1 \\
\hline Young's modulus, $E_{h}$ & variable \\
\hline Young's modulus, $E_{v}$ & $9 \mathrm{GPa}$ \\
\hline Drained Poisson's ratio, $\nu_{h}$ & variable \\
\hline Drained Poisson's ratio, $\nu_{v}$ & 0.2 \\
\hline Undrained Poisson's ratio, $\nu_{u}$ & 0.46 \\
\hline Permeability coefficient, $\kappa$ & $1 \times 10^{-17} \mathrm{~m}^{2} /(\mathrm{Pa} \cdot \mathrm{s})$ \\
\hline Porosity, $\phi$ & 0.05 \\
\hline Fluid mass density, $\rho_{f}$ & $1111.11 \mathrm{~kg} / \mathrm{cm}^{3}$ \\
\hline Fluid bulk modulus, $K_{f}$ & $3.15 \mathrm{GPa}$ \\
\hline Solid bulk modulus, $K_{S}$ & $48.2 \mathrm{GPa}$ \\
\hline Reflection coefficient, $\Re$ & 0.2 \\
\hline Swelling coefficient, $\omega_{0}$ & $1.5 \mathrm{MPa}$ \\
\hline Solute diffusion coefficient, $D_{s}$ & $2.0 \times 10^{-9} \mathrm{~m}^{2} / \mathrm{s}$ \\
\hline Thermal expansion coefficient of the solid, $\alpha_{m}$ & $6 \times 10^{-6} \mathrm{~K}^{-1}$ \\
\hline Thermal expansion coefficient of the liquid, $\alpha_{s}$ & $2.1 \times 10^{-4} \mathrm{~K}^{-1}$ \\
\hline Molar mass of the solute, $M^{S}$ & $58.5 \mathrm{~g} / \mathrm{mol}$ \\
\hline Universal gas constant, $R$ & $8.314 \mathrm{~J} /(\mathrm{mol} \cdot \mathrm{K})$ \\
\hline Thermal diffusivity, $C_{T}$ & $1.6 \times 10^{-6} \mathrm{~K}^{-1}$ \\
\hline Thermal diffusion coefficient, $D_{T}$ & $6 \times 10^{-12} \mathrm{~m}^{2} /(\mathrm{s} \cdot \mathrm{K})$ \\
\hline Thermal osmosis coefficient $K_{T}$ & $2.1 \times 10^{-11} \mathrm{~m}^{2} /(\mathrm{s} \cdot \mathrm{K})$ \\
\hline Specific entropy, $s_{0}$ & $3686 \mathrm{~J} /(\mathrm{mol} \cdot \mathrm{K})$ \\
\hline Maximum horizontal in-situ stress, $\sigma_{H}$ & $36 \mathrm{MPa}$ \\
\hline Minimum horizontal in-situ stress, $\sigma_{h}$ & $28 \mathrm{MPa}$ \\
\hline overburden stress, $\sigma_{v}$ & $44 \mathrm{MPa}$ \\
\hline Initial pore pressure, $p_{0}$ & $20 \mathrm{MPa}$ \\
\hline Initial solute mass fraction, $C_{0}^{S}$ & 0.2 \\
\hline Initial temperature, $T_{0}$ & $370 \mathrm{~K}$ \\
\hline Drilling fluid hydraulic pressure, $p_{W}$ & $24 \mathrm{MPa}$ \\
\hline Drilling fluid solute mass faction, $C_{W}^{S}$ & 0.1 \\
\hline Drilling fluid temperature, $T_{W}$ & $330 \mathrm{~K}$ \\
\hline
\end{tabular}

of the figure is the pore pressure or the effective stress variation distribution relative to the reference variable.

It can be concluded that poroelasticity (Fig. 4 solid line) induces the pore pressure to reach an extreme value in a short time in regions close to the borehole wall, and then, the extreme pore pressure moves away from wellbore wall and decreases over time. Figs. 4-6 illustrate that the heating effect of the drilling fluid could warm formation and pore fluid, meanwhile, thermal gradient forces fluid molecules move into formation, both of these effects increase the pore pressure greatly; conversely, the cooling effect of the drilling fluid has an opposite influence, the valley of the pore pressure appears at 1.05 times well radius at 60s. Furthermore, heat resistance increases with distance into the formation, greater thermal gradient distributes near the wellbore wall, so the effective radial and tangential stresses are heavily affected in the region close to the wellbore wall (Fig. 5 through 7). Fig. 5 shows that heating effect $\left(T_{W}>T_{0}\right)$ causes the effective radial stress lower first and then become higher than that the condition without thermal effect

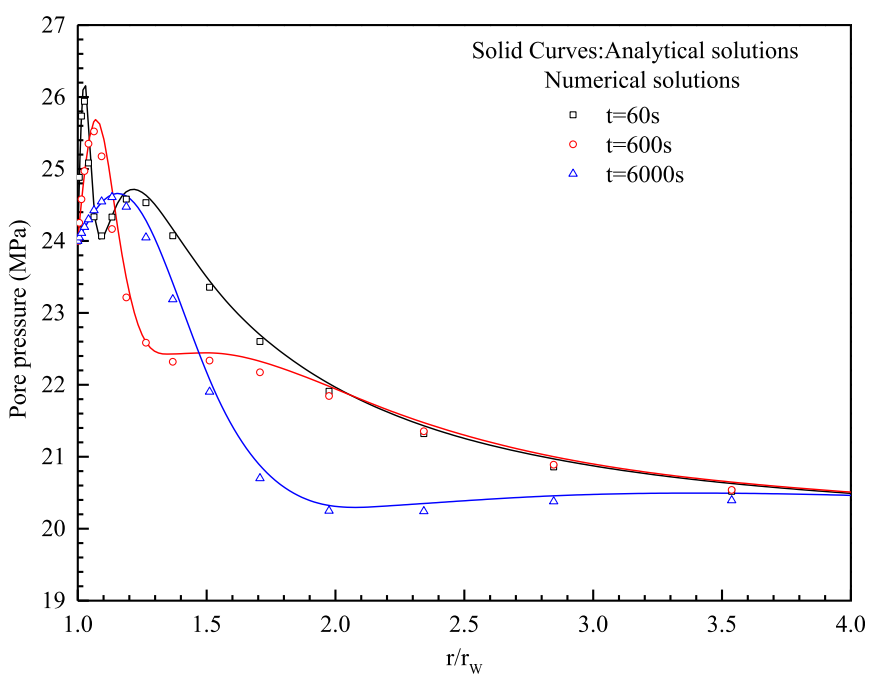

Fig. 3. Comparison of the pore pressure distribution based on chemo-thermoporoelasticity along the minimum in-situ stress direction using analytical and numerical methods. 


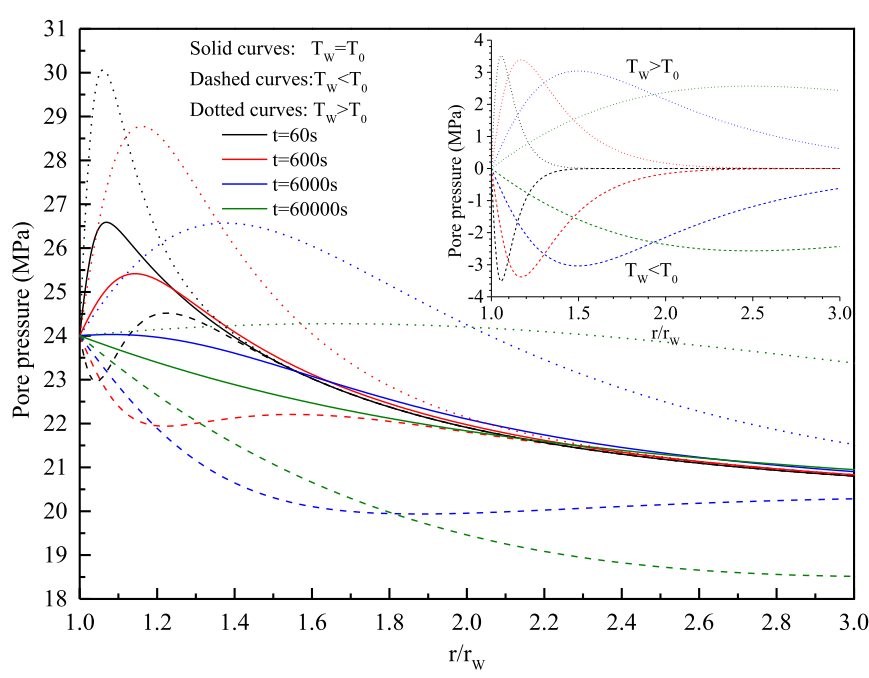

Fig. 4. Pore pressure distribution with radius along the minimum in-situ stress direction under various boundary conditions of temperature; the upper right corner is the pore pressure variation distribution under $T_{W}>T_{0}$ and $T_{W}<T_{0}$ relative to $T_{W}=T_{0}$.

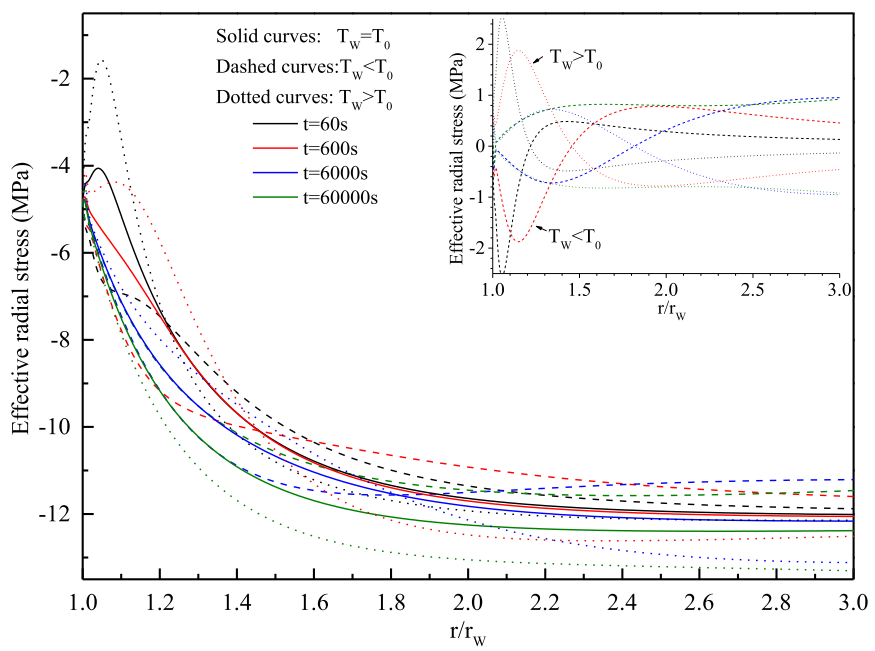

Fig. 5. Effective radial stress distribution with radius along the minimum in-situ stress direction under various boundary conditions of temperature; the upper right corner is the effective radial stress variation distribution under $T_{W}>T_{0}$ and $T_{W}<T_{0}$ relative to $T_{W}=T_{0}$.

$\left(T_{W}=T_{0}\right)$ with radial distance along the minimum in-situ stress direction, the reason is that the effective radial stress is mainly affected by thermal gradient effect on molecular diffusion of the fluid particle near the wellbore wall and by direct thermal effect far from the wellbore wall, the effective tangential stress has a similar distribution too, therefore, the heating effect could cause a higher shear stress along the minimum in-situ stress direction and the wellbore tends to fail easily around the well. As a result, a lower drill fluid temperature can help make the wellbore more stable by avoiding shear failure; however, Fig. 7 shows that the cooling effect decreases the effective tangential stress and even causes the effective tangential stress to be positive with the passage of time along the maximum in-situ stress direction, so that tensile induced fractures can occur close to the wellbore wall.

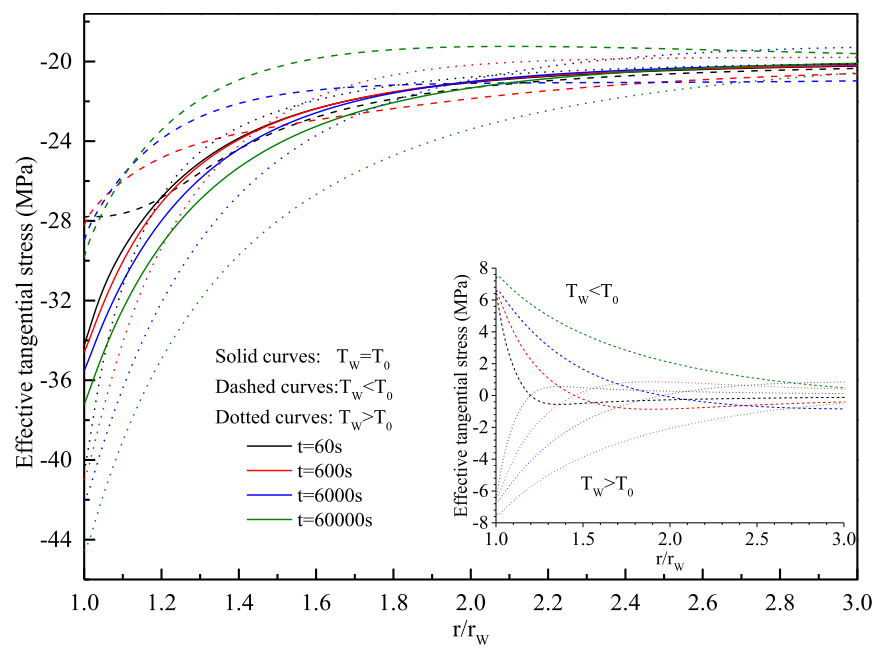

Fig. 6. Effective tangential stress distribution with radius along the minimum in-situ stress direction under various boundary conditions of temperature; the lower right corner is the effective tangential stress variation distribution under $T_{W}>T_{0}$ and $T_{W}<T_{0}$ relative to $T_{W}=T_{0}$.

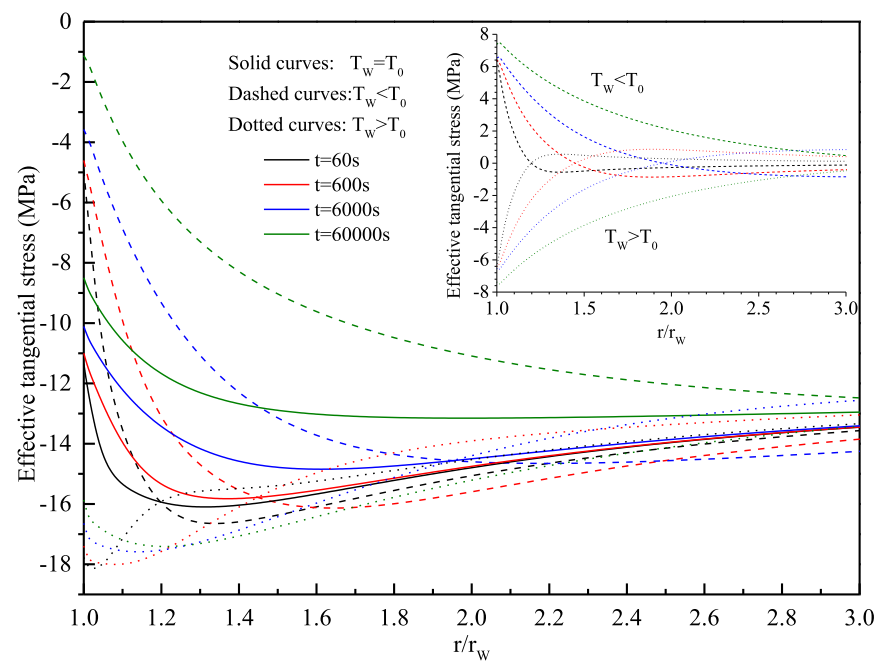

Fig. 7. Effective tangential stress distribution with radius along maximum in-situ stress direction under various boundary conditions of temperature, where the cooling effect causes the effective tangential stress to tend to be positive (tensile); the upper right corner is the effective tangential stress variation distribution under $T_{W}>T_{0}$ and $T_{W}<T_{0}$ relative to $T_{W}=T_{0}$.

Sensitivity analyses on the thermal osmosis coefficient $K_{T}$, thermal diffusion coefficient $D_{T}$ and thermal diffusivity $C_{T}$ are conducted while keeping these coefficients vary within the same order of magnitude, the temperature boundary condition is $T_{W}>T_{0}$. The transport governing equations (Eq. (7) and (8)) state that thermal osmosis coefficient $K_{T}$ affects pore fluid motion while thermal diffusion coefficient $D_{T}$ affects chemical solute diffusion and thermal diffusivity $C_{T}$ determines the distribution of temperature. The results indicate that the thermal diffusion coefficient has nearly no effect on the pore pressure (Fig. 8); the effective stresses change slightly as well, although the corresponding figures are not given due to space limitations. The reason for this observation is that temperature distribution won't be affected and the solute mass fraction is only changed very little when the thermal diffusion 


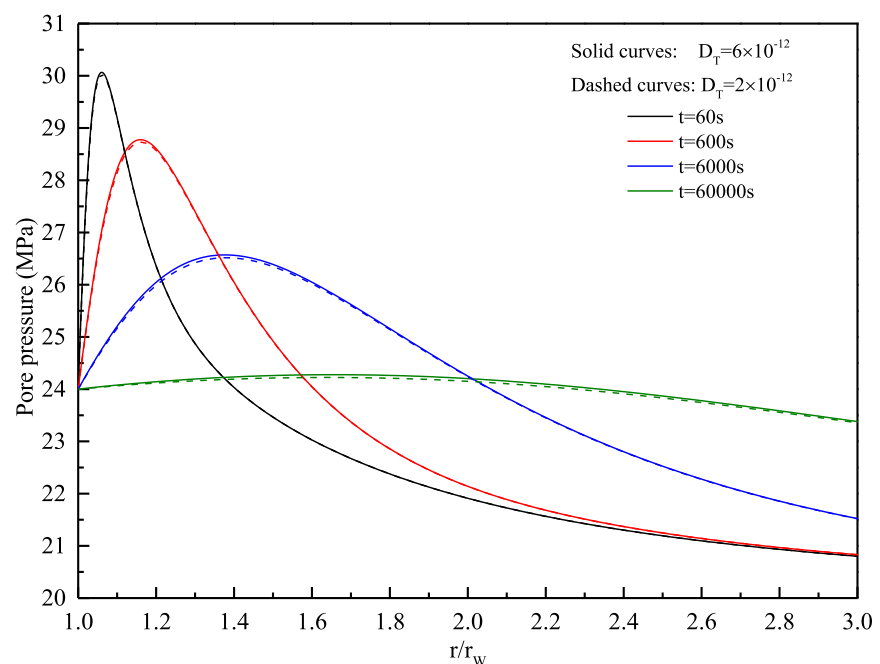

Fig. 8. Pore pressure distribution with radius along the minimum in-situ stress direction under different thermal diffusion coefficients.

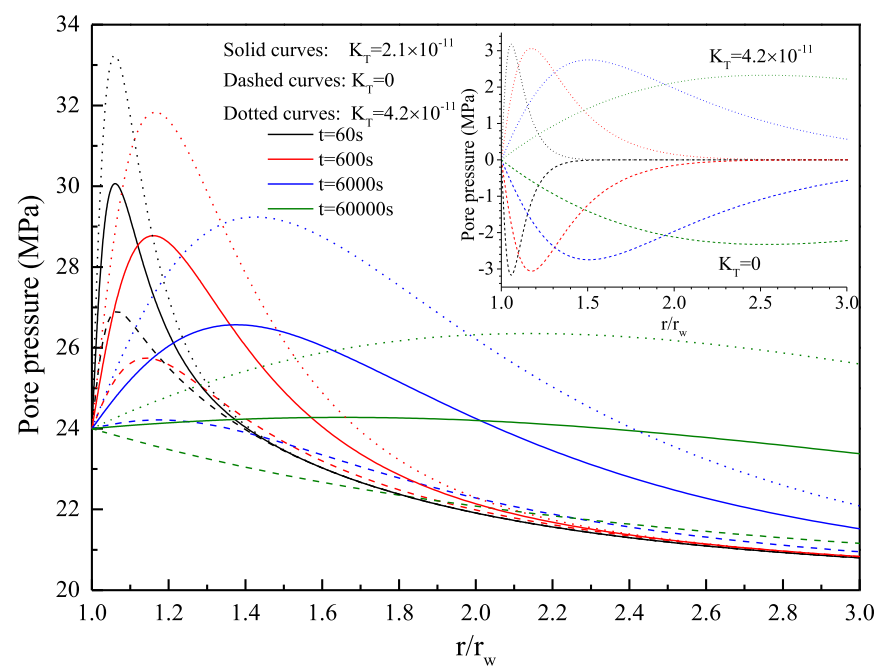

Fig. 9. Pore pressure distribution with radius along the minimum in-situ stress direction under different thermal osmosis coefficients; the upper right corner is the pore pressure variation distribution under $K_{T}=0$ and $K_{T}=4.2 \times 10^{-11} \mathrm{~m}^{2} /(\mathrm{s} \cdot K)$ relative to $K_{T}=2.1 \times 10^{-11} \mathrm{~m}^{2} /(\mathrm{s} \cdot K)$.

coefficient varies in the same order of magnitude.

Three different thermal osmosis coefficients $K_{T}$ are compared to observe its influence on the pore pressure and effective stresses, one of the coefficient values is zero, which means neglecting thermal effect on pore fluid transport (Eq. (7)). Fig. 9 shows that thermal osmosis coefficient affects the pore pressure greatly, the higher coefficient represents stronger molecular diffusion ability under same temperature gradient, Figs. 10 and 11 are effective radial and tangential stresses distribution, the result shows that radial tensile failure could happen since the effective radial stress has become positive when $K_{T}=4.2 \times 10^{-11} \mathrm{~m}^{2} /(\mathrm{s} \cdot \mathrm{K})$.

Thermal diffusivity $C_{T}$ affects temperature distribution by changing thermal diffusion, meanwhile, newer temperature gradient affect solute mass fraction and pore fluid transport, the combined coupling effect causes pore pressure decreases with higher thermal diffusivity around the wellbore (Fig. 12), lower pore pressure caused higher effective radial and tangential stresses

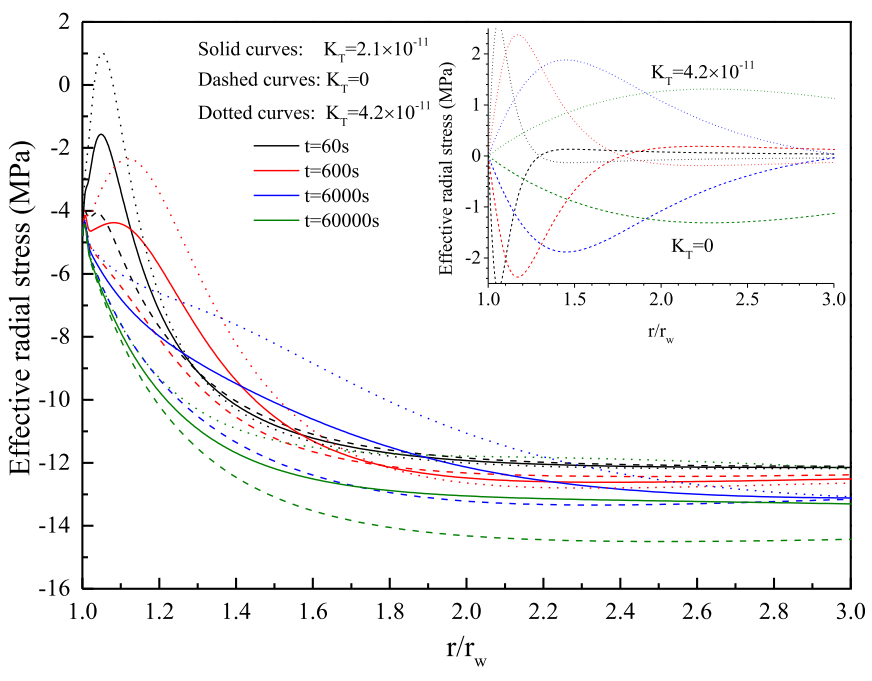

Fig. 10. Effective radial stress distribution with radius along the minimum in-situ stress direction under different thermal osmosis coefficients; the upper right corner is the effective radial stress variation distribution under $K_{T}=0$ and $K_{T}=4.2 \times 10^{-11} \mathrm{~m}^{2} /(\mathrm{s} \cdot \mathrm{K})$ relative to $K_{T}=2.1 \times 10^{-11} \mathrm{~m}^{2} /(\mathrm{s} \cdot \mathrm{K})$.

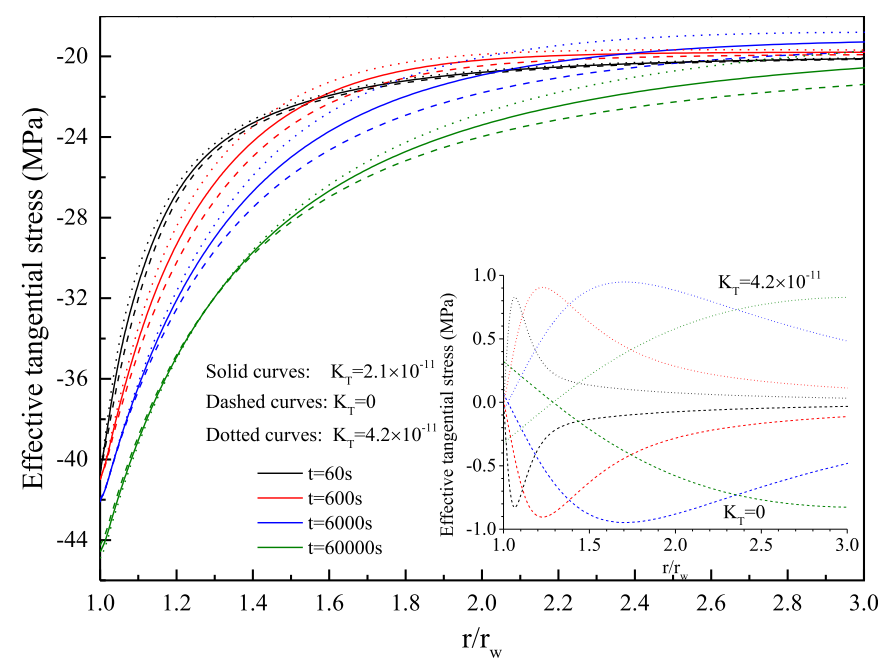

Fig. 11. Effective tangential stress distribution with radius along the minimum in-situ stress direction under different thermal osmosis coefficients; the lower right corner is the effective tangential stress variation distribution under $K_{T}=0$ and $K_{T}=4.2 \times 10^{-11} \mathrm{~m}^{2} /(\mathrm{s} \cdot \mathrm{K})$ relative to $K_{T}=2.1 \times 10^{-11} \mathrm{~m}^{2} /(\mathrm{s} \cdot \mathrm{K})$.

(Figs. 13 and 14). The result illustrates that temperature gradient effect plays a more important role on pore pressure than that of direct thermal effect.

\subsection{Effect of the solute mass fraction and chemical parameters}

The same method is presented to discuss the effects of the solute mass fraction and chemical parameters. Three cases $\left(C_{W}<C_{0}, C_{W}=C_{0}\right.$ and $\left.C_{W}>C_{0}\right)$ are still presented to investigate the pore pressure and effective stresses, the difference of solute mass fraction is assumed to be 0.1 , and the case of $C_{W}=C_{0}$ is regarded as a reference. Because the lower solute mass fraction of the drilling fluid causes water to invade the formation more easily, as shown in Figs. 15 and 16, the lower solute mass fraction of the drilling fluid increases the pore pressure and decreases the effective radial stresses rapidly in a short time close to the wellbore wall. When 


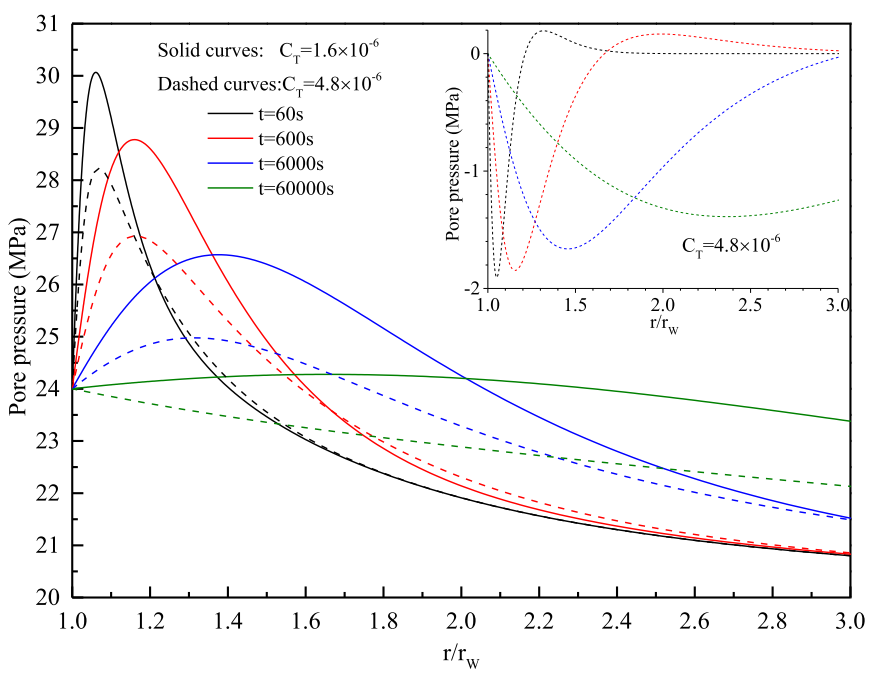

Fig. 12. Pore pressure distribution with radius along the minimum in-situ stress direction under different thermal diffusivity values; the upper right corner is the pore pressure variation distribution under $C_{T}=4.8 \times 10^{-6} \mathrm{~K}^{-1}$ relative to $C_{T}=1.6 \times 10^{-6} K^{-1}$.

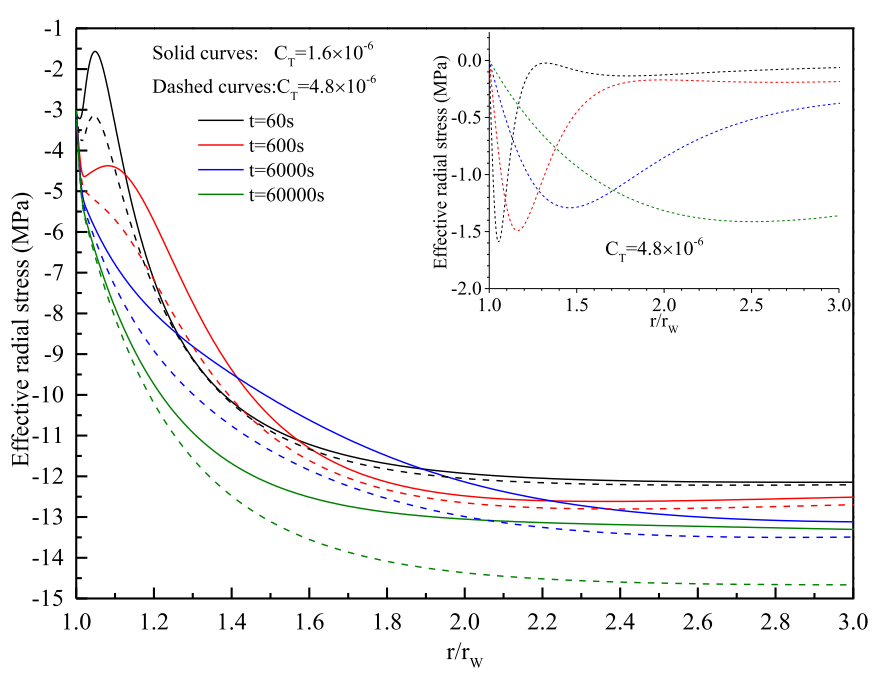

Fig. 13. Effective radial stress distribution with radius along the minimum in-situ stress direction under different thermal diffusivity; the upper right corner is the effective radial stress variation distribution under $C_{T}=4.8 \times 10^{-6} \mathrm{~K}^{-1}$ relative to $C_{T}=1.6 \times 10^{-6} \mathrm{~K}^{-1}$.

$C_{W}>C_{0}$, the dotted line in Fig. 15 indicates that the pore pressure decreases close to the wellbore wall, that is, chemical osmosis has overcome the hydraulic gradient and forces fluid out of the formation. It can be concluded that the pore pressure is most sensitive to the chemical effect, whereas the effective tangential stress (Fig. 17) is least sensitive to the chemical effect, which is contrast to the thermal effect. When $C_{W}<C_{0}$, the short-term effective radial stress decreases rapidly; one consequence is that the shear failure risk may increase, and another consequence is that the chemical effect causes the effective radial stress tend to be less compressive. As a result, wellbore "spalling" instability may occur when the effective radial stress is positive. Thus, keeping the solute concentration of the drilling fluid higher than that of the pore fluid is beneficial for wellbore stability.

Sensitivity analysis on the chemical parameters is conducted by

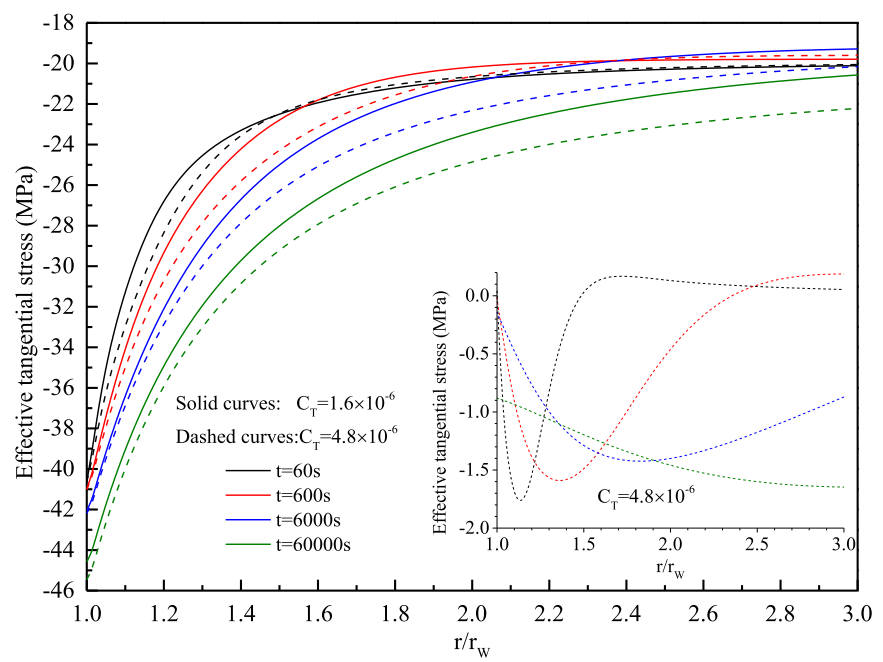

Fig. 14. Effective tangential stress distribution with radius along the minimum in-situ stress direction under different thermal diffusivity; the lower right corner is the effective tangential stress variation distribution under $C_{T}=4.8 \times 10^{-6} \mathrm{~K}^{-1}$ relative to $C_{T}=1.6 \times 10^{-6} K^{-1}$.

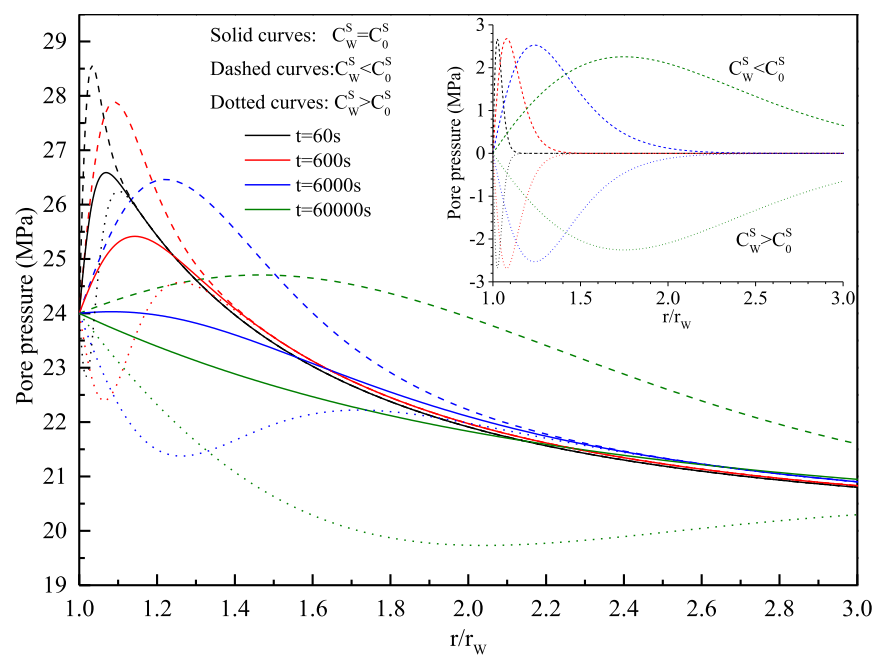

Fig. 15. Pore pressure distribution with radius along the minimum in-situ stress direction under various boundary conditions of the solute mass fraction; the upper right corner is the pore pressure variation distribution under $C_{W}^{S}>C_{0}^{S}$ and $C_{W}^{S}<C_{0}^{S}$ relative to $C_{W}^{S}=C_{0}^{S}$.

changing the reflection coefficient $\Re$ and the solute diffusion coefficient $D_{S}$ within the same order of magnitude; the analysis is conducted assuming $C_{W}>C_{0}$.and $T_{W}=T_{0}$, and two cases ( $\Re=0.1$ and $\Re=0.3, D_{S}=2 \times 10^{-9}$ and $D_{S}=6 \times 10^{-9}$ ) are presented to investigate the effect of the chemical parameters; the reference, which is the condition of $\Re=0.1$ and $D_{S}=2 \times 10^{-9}$, is used to observe the variations when increasing the chemical parameters. Figs. 18-20 illustrate that the pore pressure and effective stress are greatly influenced by the reflection coefficient: a higher reflection coefficient decreases the pore pressure and increases the effective stresses. The reason for this behavior is that the reflection coefficient reflects the membrane efficiency of formation, which is a chemical coupling parameter in the fluid transport equation (Eq. (8)): a high reflection coefficient value denotes a better membrane efficiency, which can keep chemical osmosis stable and prevents solutes from moving into or out of the formation. 


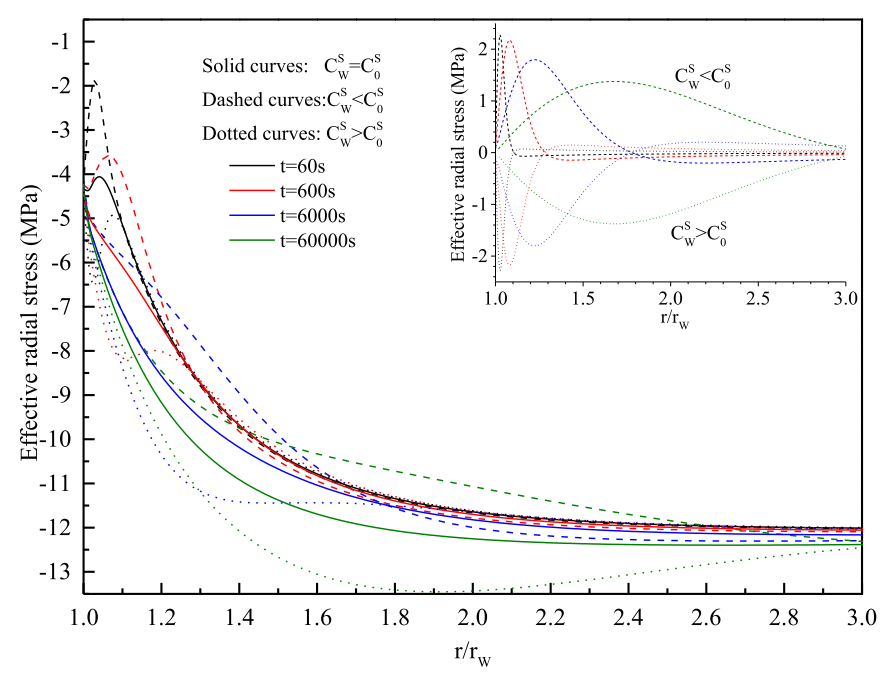

Fig. 16. Effective radial stress distribution with radius along the minimum in-situ stress direction under various boundary conditions of the solute mass fraction; the upper right corner is the effective radial stress variation distribution under $C_{W}^{S}>C_{0}^{S}$ and $C_{W}^{S}<C_{0}^{S}$ relative to $C_{W}^{S}=C_{0}^{S}$.

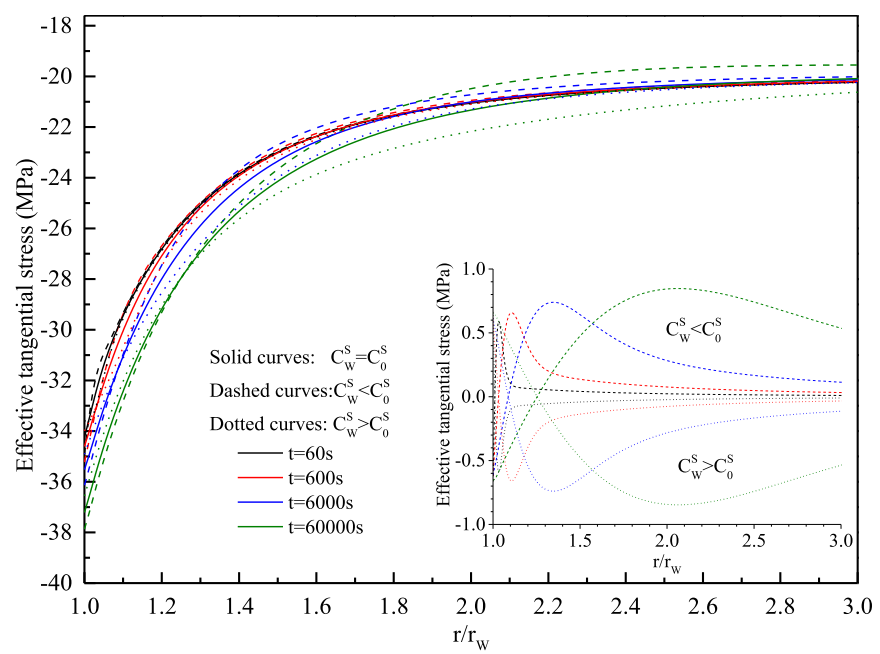

Fig. 17. Effective tangential stress distribution with radius along the minimum in-situ stress direction under various boundary conditions of the solute mass fraction; the lower right corner is the effective tangential variation distribution under $C_{W}^{S}>C_{0}^{S}$ and $C_{W}^{S}<C_{0}^{S}$ relative to $C_{W}^{S}=C_{0}^{S}$.

A high solute diffusion coefficient $\left(D_{S}\right)$ value could improve solute transport and reduce the chemical gradient between the drilling fluid and pore fluid; thus, the effect of a higher solute diffusion coefficient is the same as that of a lower reflection coefficient, but the analysis results show that the pore pressure and effective stresses are less sensitive to solute diffusion than the reflection coefficient in the same order of magnitude (Figs. 21-23).

Fig. 24 shows the pore pressure distribution around the well along the minimum in-situ stress direction after $600 \mathrm{~s}$ when taking into account the combining effects of the thermal and chemical loadings; the lower temperature and higher solute mass fraction of the drilling fluid cause the lowest pore pressure, which is beneficial for wellbore stability. Moreover, when $C_{W}^{S}>C_{0}^{S}, T_{W}>T_{0}$, the pore pressure grows first higher and then becomes lower than the condition of $C_{W}^{S}<C_{0}^{S}, T_{W}<T_{0}$ when the region is farther than 1.1 times wellbore radius, that is, thermal osmosis has a greater effect

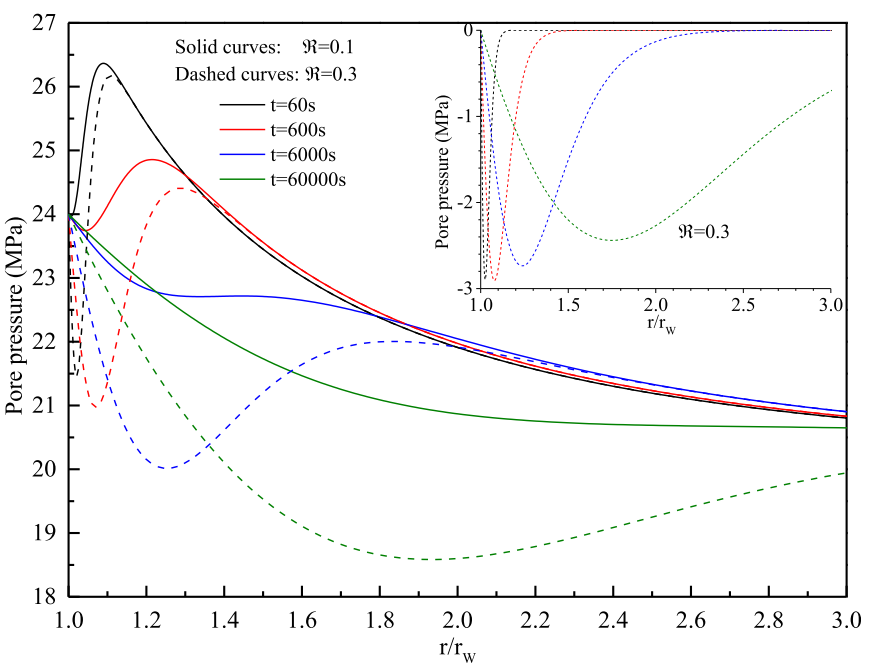

Fig. 18. Pore pressure distribution with radius along the minimum in-situ stress direction under different reflection coefficients; the upper right corner is the pore pressure variation distribution under $\Re=0.3$ relative to $\Re=0.1$.

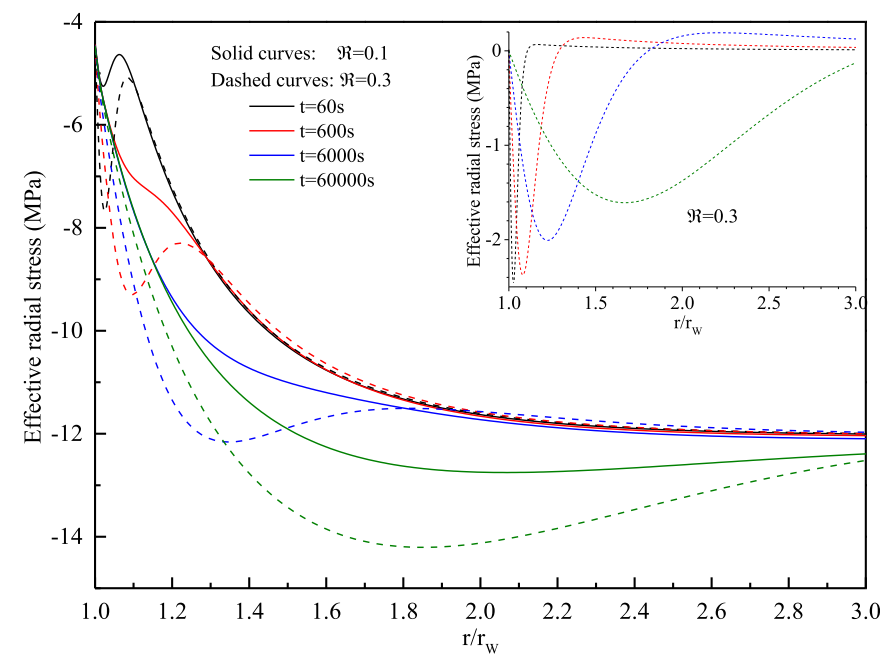

Fig. 19. Effective radial stress with radius along the minimum in-situ stress direction under different reflection coefficients; the upper right corner is the effective radial stress variation distribution under $\Re=0.3$ relative to $\Re=0.1$.

than chemical osmosis in the region close to the wellbore wall. Hence, it is a recommended practice to ensure the drilling fluid solute concentration is higher and temperature is lower than that of the pore fluid to balance the pore pressure and maintain wellbore stability.

\subsection{Effect of the anisotropy ratios}

Material constants, such as Young's modulus, Poisson's ratio and permeability along the horizontal and vertical directions of a bedding plane are different for a transverse isotropic formation; to study the effect of rock anisotropy ratios on the pore pressure and effective stresses, a directional well drilled in horizontal bedding formation is assumed, with an inclination of $45^{\circ}$ and the azimuth direction along the maximum in-situ stress direction.

Three cases are discussed to study the sensitivity of Young's modulus, Poisson's ratio and permeability, with one of these cases 


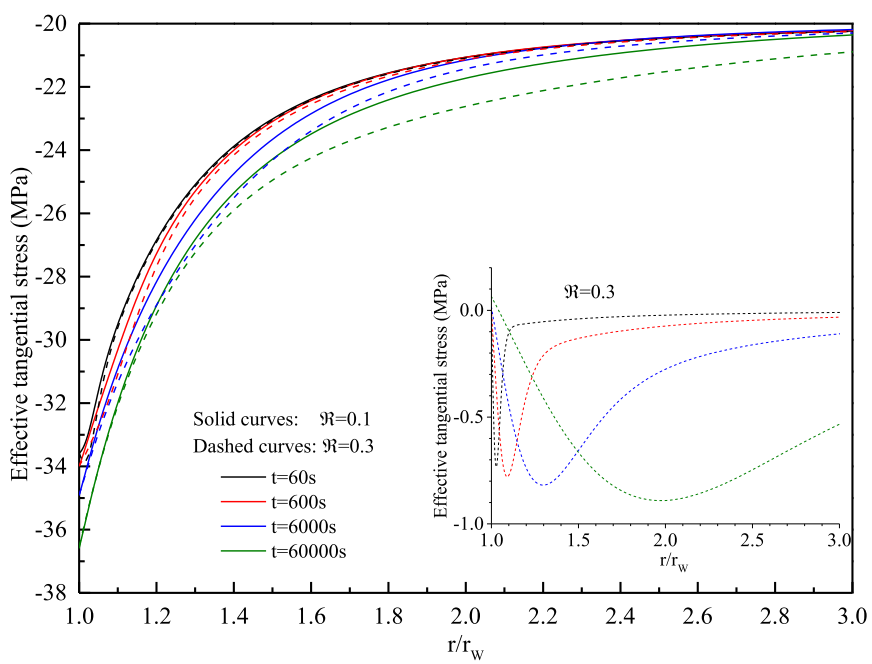

Fig. 20. Effective tangential stress with radius along the minimum in-situ stress direction under different reflection coefficients; the lower right corner is the effective tangential stress variation distribution under $\Re=0.3$ relative to $\Re=0.1$.

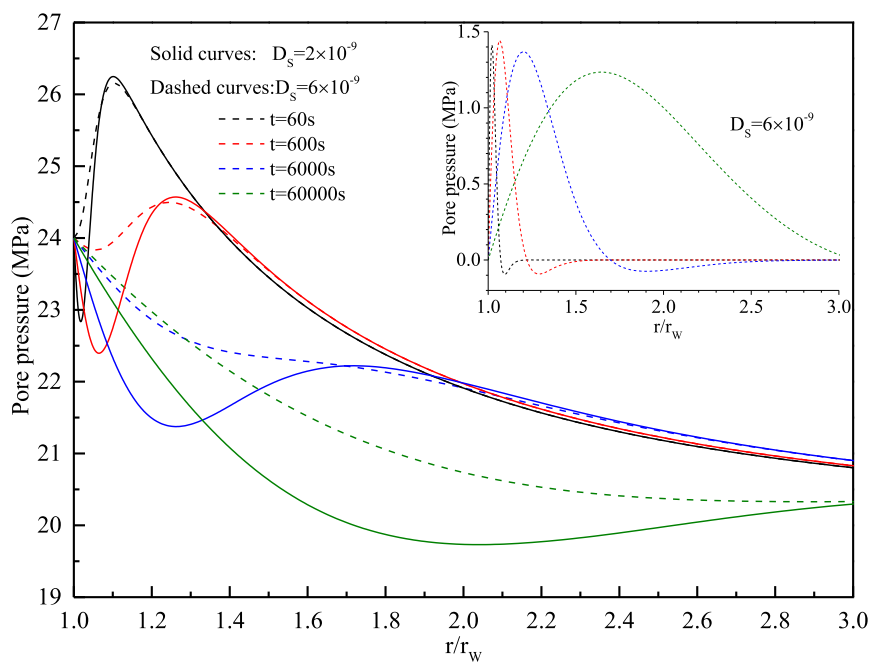

Fig. 21. Pore pressure distribution with radius along the minimum in-situ stress direction under different solute diffusion coefficients; the upper right corner is the pore pressure variation distribution under $D_{S}=6 \times 10^{-9}$ relative to $D_{S}=2 \times 10^{-9}$.

being an isotropic formation that serves as a reference. The anisotropy ratio is defined as the ratio of the horizontal material constant to vertical material constants. The general effect of transverse isotropy ratios is that a higher Young's modulus or lower Poisson's ratio increases pore pressure in the region to the wellbore wall, Constitutive equations (Eq. (1) and (2)) show that several coefficient tensors $\left(\mathbf{D}, \gamma_{1}\right)$ and coefficients $\left(\beta, \chi^{\prime}, \gamma_{2}\right)$ are related to elastic properties, especially the drained bulk modulus of elasticityK, and shear modulus $G$, therefore, different Young's modulus and Poisson's ratios have a combined and complicate effect on pore pressure and effective stresses distribution. Different Young's modulus ratios could greatly change the pore pressure and effective stresses: a higher Young's modulus ratio reduces the pore pressure and effective tangential stress, but increases the effective radial stress significantly in regions close to the wellbore wall (Figs. 25-27). However, different horizontal and vertical Poisson's ratios have very slight effects on the pore pressure (Fig. 28); the

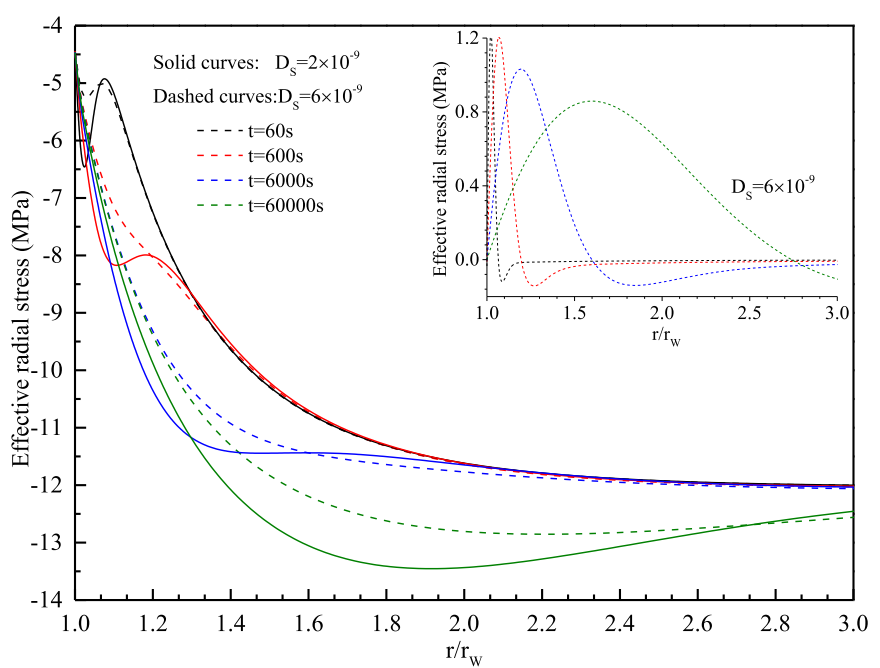

Fig. 22. Effective radial stress distribution with radius along the minimum in-situ stress direction under different solute diffusion coefficients; the upper right corner is the effective radial stress variation distribution under $D_{S}=6 \times 10^{-9}$ relative to $D_{S}=2 \times 10^{-9}$

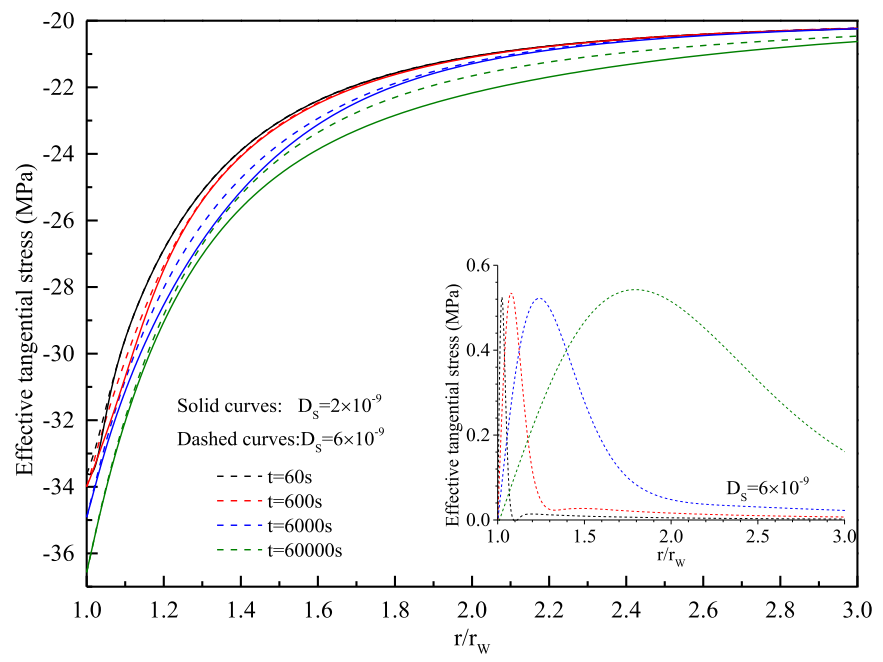

Fig. 23. Effective tangential stress distribution with radius along the minimum in-situ stress direction under different solute diffusion coefficients; the lower right corner is the effective tangential stress variation distribution under $D_{S}=6 \times 10^{-9}$ relative to $D_{S}=2 \times 10^{-9}$

pore pressure variations under $\nu_{h}=0.5 v_{v}$ and $\nu_{h}=2.0 v_{v}$ relative to $\nu_{h}=\nu_{v}$ are less than 1.0 MPa. The effective radial and tangential stress variations are less than $1.3 \mathrm{MPa}$; thus, the stress distribution figures are not shown here due to space limitations.

The permeability along the isotropic bedding plane is generally higher than that along the perpendicular direction; thus, three cases $\left(k_{h}=k_{v}, k_{h}=3 k_{v}\right.$ and $\left.k_{h}=9 k_{v}\right)$ are presented together to study the effect of the permeability anisotropic ratio, with the result of $k_{h}=k_{v}$ regarded as the reference. The higher permeability ratio greatly decreases the pore pressure and thus increases the effective radial stresses and tangential stresses, particularly in regions close to the wellbore wall (Figs. 29-31). Lower permeability could hinder hydraulic diffusion and is not beneficial for increasing pore pressure, however, it is more difficult for pore fluid to drain 


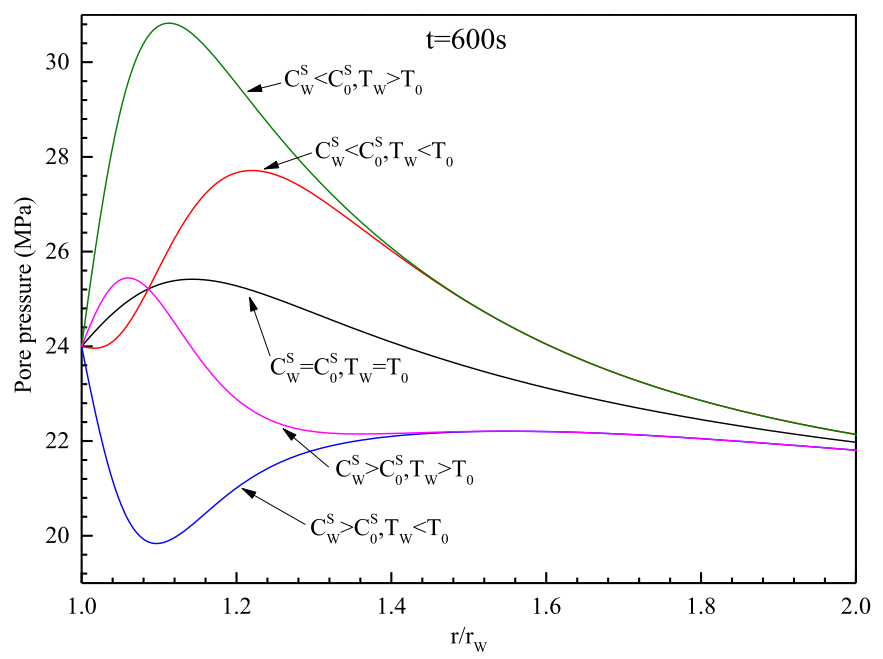

Fig. 24. Pore pressure distribution along the minimum in-situ stress direction under different boundary conditions of temperature and solute mass fraction after $600 \mathrm{~s}$.

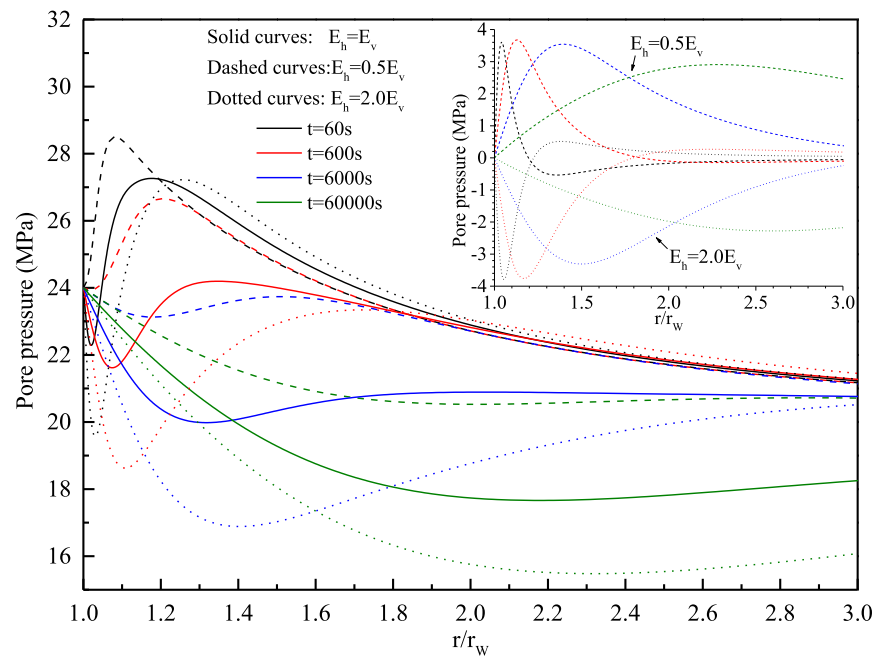

Fig. 25. Pore pressure distribution with radius along the minimum in-situ stress direction under different Young's modulus ratios; the upper right corner is the pore pressure variations distribution under $E_{h}=0.5 E_{v}$ and $E_{h}=2.0 E_{v}$ relative to $E_{h}=E_{v}$.

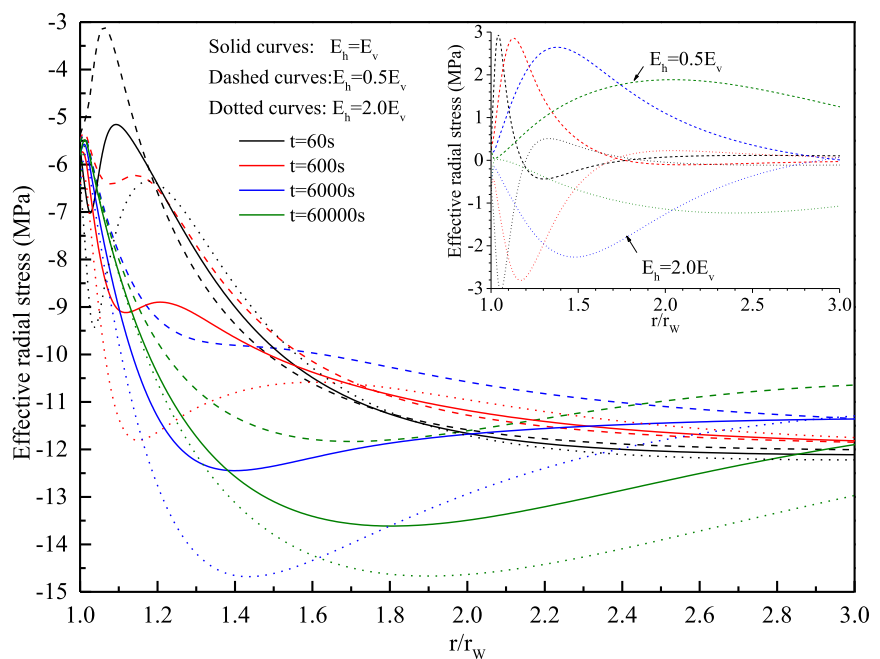

Fig. 26. Effective radial stress distribution with radius along the minimum in-situ stress direction under different Young's modulus ratios; the upper right corner is the effective radial stress variations distribution under $E_{h}=0.5 E_{v}$ and $E_{h}=2.0 E_{v}$ relative to $E_{h}=E_{v}$.

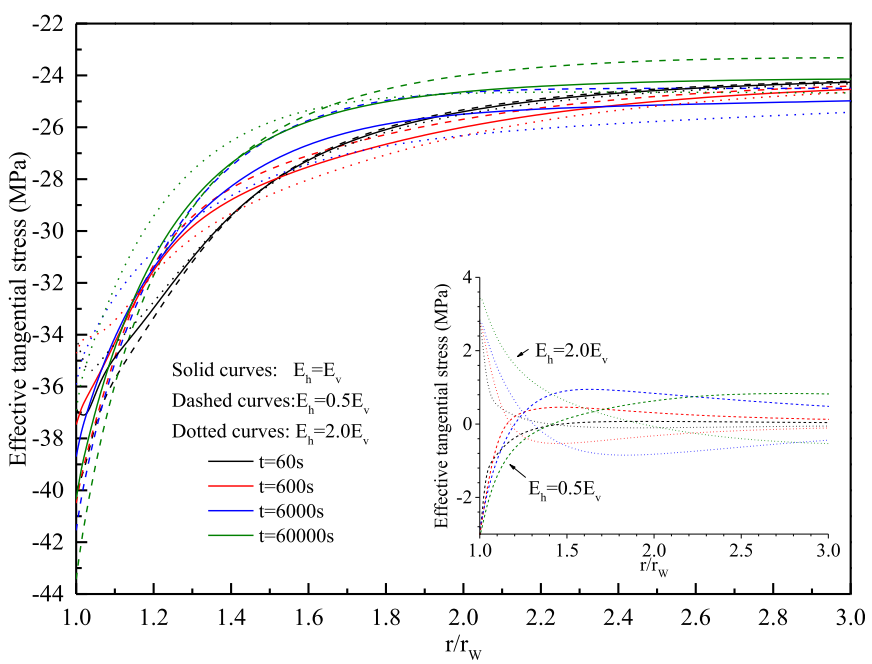

Fig. 27. Effective tangential stress distribution with radius along the minimum in-situ stress direction under different Young's modulus ratios; the lower right corner is the effective tangential stress variations distribution under $E_{h}=0.5 E_{v}$ and $E_{h}=2.0 E_{v}$ relative to $E_{h}=E_{v}$.

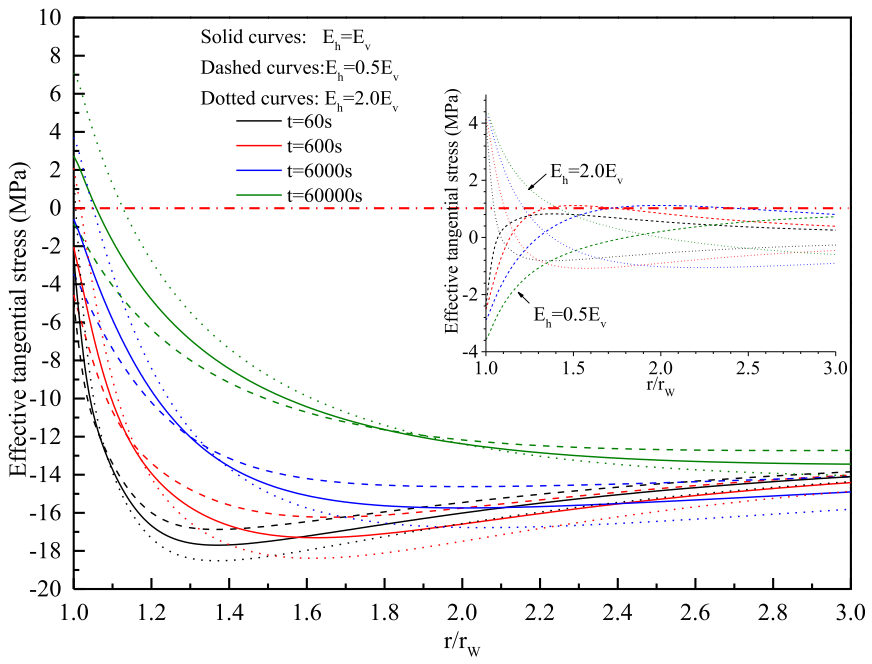

Fig. 28. Pore pressure distribution with radius along the minimum in-situ stress direction under different Poisson's ratios; the upper right corner is the pore pressure variations distribution under $\nu_{h}=0.5 v_{v}$ and $\nu_{h}=2.0 v_{v}$ relative to $\nu_{h}=\nu_{v}$.

from pore space in lower permeability shale, which could enhance the Skempton effect (Ekbote, 2002) that causes an excess pore pressure because of coupled deformation of pore fluid and solid in stress concentration area, this Skempton effect could dominate the pore pressure distribution while the order of magnitude of shale permeability is very low. The results imply that the permeability magnitude and anisotropic ratio are critical for the pore pressure and effective stress distributions.

\section{Conclusion}

A fully coupled chemo-thermo-poroelastic non-linear model was developed to analyze the pore pressure and effective stresses distribution around a wellbore using the finite element method. The model is available for use in an arbitrary direction well in a transversely isotropic formation; as a result, this model addresses the application limitations of the analytical solutions.

The model is used to study the impact of temperature and solute 


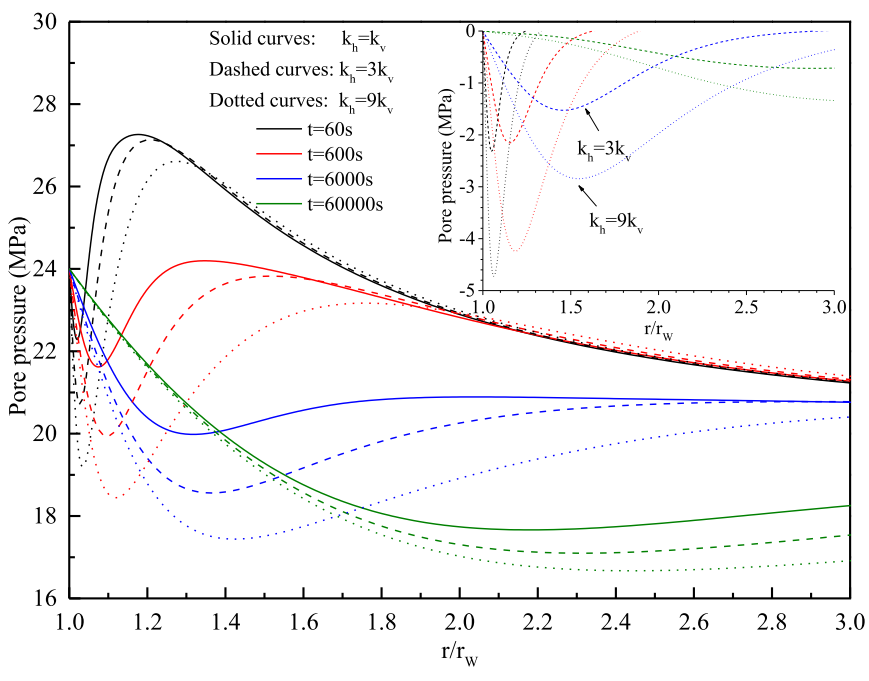

Fig. 29. Pore pressure distribution with radius along the minimum in-situ stress direction under different permeability ratios; the upper right corner is the pore pressure variations distribution under $k_{h}=3 k_{v}$ and $k_{h}=9 k_{v}$ relative to $k_{h}=k_{v}$.

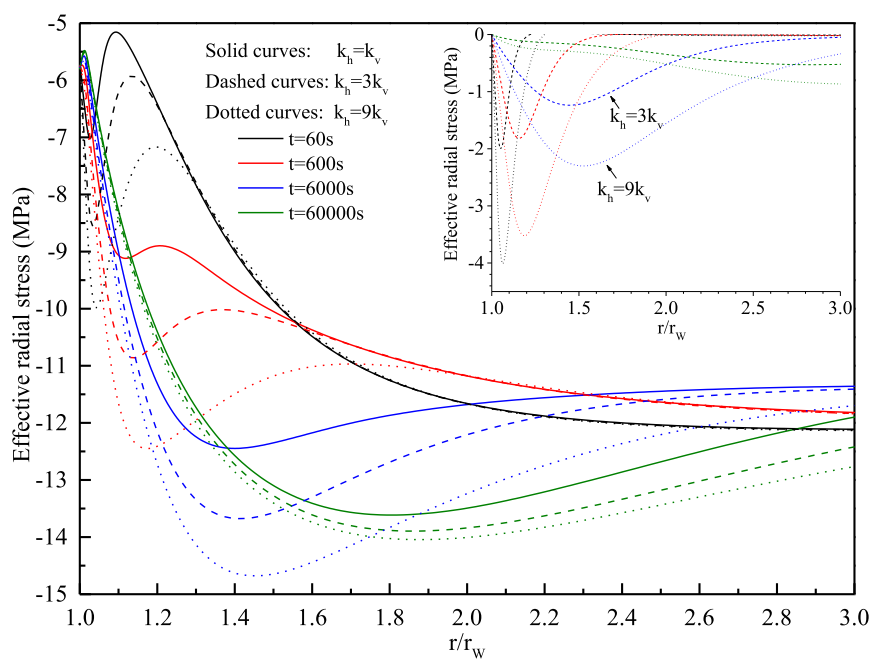

Fig. 30. Effective radial stress distribution with radius along the minimum in-situ stress direction under different permeability ratios; the upper right corner is the effective radial stress variations distribution under $k_{h}=3 k_{v}$ and $k_{h}=9 k_{v}$ relative to $k_{h}=k_{v}$.

mass fraction of drilling fluid on the pore pressure and effective stresses around a well; the results indicate that a lower temperature of the drilling fluid decreases the pore pressure and effective stresses and is therefore beneficial to wellbore stability; however, because the effective tangential stress is most sensitive to temperature, tensile-induced fractures are easily formed because of the cooling effect of the drilling fluid. Thermal osmosis has more critical effect on the pore pressure and effective stresses than that of chemical osmosis in the region close to the wellbore wall. As expected: a higher solute mass fraction could greatly decrease the pore pressure and maintain wellbore stability.

When an inclined well is drilled in a transversely isotropic shale formation, sensitivity analysis reveals that the Young's modulus and permeability anisotropy ratio significantly change the pore pressure and effective stresses, whereas Poisson's ratio has a slight effect on them.

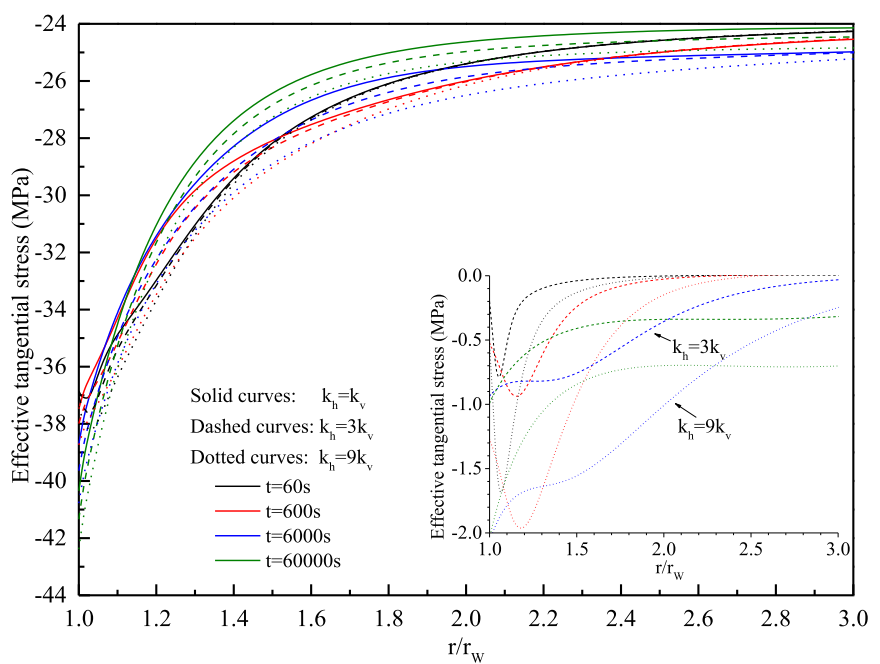

Fig. 31. Effective tangential stress distribution with radius along the minimum in-situ stress direction under different permeability ratios; the lower right corner is the effective tangential stress variations distribution under $k_{h}=3 k_{v}$ and $k_{h}=9 k_{v}$ relative to $k_{h}=k_{v}$

\section{Acknowledgements}

The authors gratefully acknowledge the financial support from the Natural Science Foundation of China (No. 11502304, No.51521063), the National Basic Research Program of China (2015CB251202) and the Science Foundation of China University of Petroleum, Beijing (No. C201601, No. 2462013YJRC023). The UEL subroutine code of chemo-thermo-poroelasticity used in this paper is based on poroelasticity code written by Dr. Wei Liu.

\section{Nomenclature}

$C^{S} \quad$ solute mass fraction

$C^{D} \quad$ solvent mass fraction

D drained elastic stiffness tensor

$\mathbf{D}_{S} \quad$ solute diffusion coefficient

$\mathbf{D}_{T} \quad$ thermal diffusion coefficient

$E_{h} \quad$ Young's modulus in the isotropic plane

$E_{v} \quad$ Young's modulus in the transverse direction

k formation permeability

$K \quad$ drained bulk modulus of elasticity

$K_{f} \quad$ fluid bulk modulus

$K_{S} \quad$ solid matrix bulk modulus

$K_{T} \quad$ thermal osmosis coefficient

$\mathbf{k}^{T} \quad$ thermal conductivity

$G \quad$ shear modulus in the isotropic plane

$G^{\prime} \quad$ shear modulus in the transverse direction

$M^{s} \quad$ molar mass of the solute

$p \quad$ pore pressure

$R \quad$ universal gas constant

$s_{0} \quad$ the reference value of the specific fluid entropy at the average system temperature and solute mass fraction absolute temperature

Biot's coefficient

thermal expansion coefficients of the fluid thermal expansion coefficients of the solid strain tensor

Poisson' ratio in the isotropic plane

Poisson' ratio in the transverse direction

fluid mass density

total stress tensor 
fluid viscosity permeability coefficient formation porosity reflection coefficient

\section{Appendix}

$$
\mathbf{H}_{4}=\int_{\Omega} \mathbf{N}_{T}^{T} \cdot \mathbf{N}_{T} d V
$$

$\mathbf{Q}_{4}=\int_{\Omega} \mathbf{B}_{T}^{T} \cdot C_{T} \mathbf{B}_{T} d V$

\section{References}

Abousleiman, Y., Ekbote, S., 2001. Porothermoelasticity in transversely isotropic porous materials. In: Ehlers, W. (Ed.), IUTAM Symposium on Theoretical and Numerical Methods in Continuum Mechanics of Porous Materials, Solid Mechanics and its Applications. Springer, Netherlands, pp. 145-152. http:// dx.doi.org/10.1007/0-306-46953-7_21.

Biot, M.A., 1941. General theory of three-dimensional consolidation. J. Appl. Phys. 12, $155-164$.

Chenevert, M.E., Dewan, J.T., 2001. A model for filtration of water-base mud during drilling: determination of mudcake parameters. Petrophysics 42.

Cui, L., Cheng A.H.D., Kaliakin, V.N., Abousleiman, Y, Roegiers, J.-C., 1996. Finite element analyses of anisotropic poroelasticity: a generalized Mandel's problem and an inclined borehole problem. Int. J. Numer. Anal. Methods Geomech. 20 381-401. http://dx.doi.org/10.1002/(SICI)1096-9853(199606)20:6<381::AIDNAG826>3.0.CO;2-Y.

Cui, L., Kaliakin, V.N., Abousleiman, Y., Cheng, A.H.-D., 1997. Finite element formulation and application of poroelastic generalized plane strain problems. Int. J Rock Mech. Min. Sci. 34, 953-962. http://dx.doi.org/10.1016/S1365-1609(97) 80005-6.

Detournay, E., Cheng, A.-D., 1988. Poroelastic response of a borehole in a nonhydrostatic stress field. In: International Journal of Rock Mechanics and Mining Sciences \& Geomechanics Abstracts. Elsevier, pp. 171-182.

Diek, A., White, L., Roegiers, J.-C., Bartko, K., Chang, F., 2011. ARMA-11-406. In: A Fully Coupled Thermoporoelastic Model for Drilling in Chemically Active Formations. American Rock Mechanics Association, ARMA.

Ekbote, S., Abousleiman, Y., 2006. Porochemoelastic solution for an inclined borehole in a transversely isotropic formation. J. Eng. Mech. 132, 754-763.

Ekbote, S.M., 2002. Anisotropic Poromechanics of the Wellbore Coupled with Thermal and Chemical Gradients.

Ghassemi, A., Cheng, A.H.-D., Diek, A., Roegiers, J.-C., 2001. A complete plane strain fictitious stress boundary element method for poroelastic media. Eng. Anal. Bound. Elem. 25, 41-48. http://dx.doi.org/10.1016/S0955-7997(00)00046-1.

Ghassemi, A., Diek, A., 2003. Linear chemo-poroelasticity for swelling shales: theory and application. J. Pet. Sci. Eng. Boreh. Stab. 38, 199-212. http://dx.doi.org/ 10.1016/S0920-4105(03)00033-0.

Ghassemi, A., Diek, A., 2002. SPE-78162-MS. In: A Chemo-poroelastic Solution for Stress and Pore Pressure Distribution Around a Wellbore in Transversely Isotropic Shale. Society of Petroleum Engineers, SPE. http://dx.doi.org/10.2118 78162-MS.

Ghassemi, A., Tao, Q., Diek, A., 2009. Influence of coupled chemo-porothermoelastic processes on pore pressure and stress distributions around a wellbore in swelling shale. J. Pet. Sci. Eng. 67, 57-64. http://dx.doi.org/10.1016 j.petrol.2009.02.015.

Ghassemi, A., Wolfe, G., Diek, A., Roegiers, J.-C., 1999. ARMA-99-0239. In: A Chemomechanical Model for Borehole Stability Analyses. American Rock Mechanics Association, ARMA.

Ghassemi, A., Zhang, Q., 2004. A transient fictitious stress boundary element method for porothermoelastic media. Eng. Anal. Bound. Elem. 28, 1363-1373. http://dx.doi.org/10.1016/j.enganabound.2004.05.003.

Gomar, M., Goodarznia, I., Shadizadeh, S.R., 2015. Transient thermo-poroelastic analysis of drilling-induced mechanical damage in nonfractured rocks. Arab. J. Geosci. 1-16. http://dx.doi.org/10.1007/s12517-015-1950-0.

Gomar, M., Goodarznia, I., Shadizadeh, S.R., 2014a. A transient fully coupled thermoporoelastic finite element analysis of wellbore stability. Arab. J. Geosci. 8, 3855-3865. http://dx.doi.org/10.1007/s12517-014-1461-4.

Gomar, M., Goodarznia, I., Shadizadeh, S.R., 2014b. Transient thermo-poroelastic finite element analysis of borehole breakouts. Int. J. Rock Mech. Min. Sci. 71, 418-428. http://dx.doi.org/10.1016/j.ijrmms.2014.08.008.

Heidug, W.K., Wong, S.-W., 1996. Hydration swelling of water-absorbing rocks: a constitutive model. Int. J. Numer. Anal. Methods Geomech. 20, 403-430. http:/ dx.doi.org/10.1002/(SICI)1096-9853(199606)20:6<403::AIDNAG832>3.0.CO;2-7.

Hibbett, Karlsson, Sorensen, 1998. ABAQUS/standard: User's Manual. Hibbitt Karlsson \& Sorensen.

Lekhnitskii, S.G., 1977. Elasticity Theory of Anisotropic Bodies. Nauka, Moscow.

Mody, F.K., Hale, A.H., 1993a. Borehole-stability model to couple the mechanics and chemistry of DriUing-fluid/shale interactions. J. Pet. Technol. 1093-1101.

Mody, F.K., Hale, A.H., 1993b. Borehole-stability Model to Couple the Mechanics and Chemistry of Drilling-fluid/shale Interactions. SPE-25728-PA. http://dx.doi.org/ 10.2118/25728-PA. 
Palciauskas, V.V., Domenico, P.A., 1982. Characterization of drained and undrained response of thermally loaded repository rocks. Water Resour. Res. 18, 281-290.

Roshan, H., Aghighi, M.A., 2012. Chemo-poroelastic analysis of pore pressure and stress distribution around a wellbore in swelling shale: effect of undrained response and horizontal permeability anisotropy. Geomech. Geoengin. 7, 209-218. http://dx.doi.org/10.1080/17486025.2011.616936.

Sherwood, J.D., 1993. Biot poroelasticity of a chemically active shale. Proc. R. Soc Lond. Math. Phys. Eng. Sci. 440, 365-377. http://dx.doi.org/10.1098/ rspa.1993.0021.

Sherwood, J.D., Craster, B., 2000. Transport of water and ions through a clay membrane. J. Colloid Interface Sci. 230, 349-358. http://dx.doi.org/10.1006/ jcis. 2000.7100

Tao, Q., Ghassemi, A., 2006. Optimization of Mud Properties for Drilling in Shale Using Coupled Chemo-poro-thermoelasticity. Presented at the Golden Rocks
2006, The 41st U.S. Symposium on Rock Mechanics (USRMS). American Rock Mechanics Association.

Tao, Q., Ghassemi, A., Diek, A., 2005. ARMA-05-726. In: A Chemo-poro-thermoelastic Model for Stress/Pore Pressure Analysis Around a Wellbore in Shale. American Rock Mechanics Association, ARMA.

Wang, Y., Dusseault, M.B., 2003. A coupled conductive-convective thermoporoelastic solution and implications for wellbore stability. J. Pet. Sci. Eng. Boreh. Stab. 38, 187-198. http://dx.doi.org/10.1016/S0920-4105(03)00032-9.

Wang, Y., Papamichos, E., 1994. Conductive heat flow and thermally induced fluid flow around a well bore in a poroelastic medium. Water Resour. Res. 30, 3375-3384. http://dx.doi.org/10.1029/94WR01774.

Zhou, X., Ghassemi, A., 2009. Finite element analysis of coupled chemo-porothermo-mechanical effects around a wellbore in swelling shale. Int. J. Rock Mech. Min. Sci. 46, 769-778. http://dx.doi.org/10.1016/j.ijrmms.2008.11.009. 